\title{
EFD and CFD Characterization of a CLT Propeller
}

\author{
Daniele Bertetta, ${ }^{1}$ Stefano Brizzolara, ${ }^{1}$ Edward Canepa, ${ }^{2}$ \\ Stefano Gaggero, ${ }^{1}$ and Michele Viviani ${ }^{1}$ \\ ${ }^{1}$ Department of Naval Architecture, Marine Engineering and Electrical Engineering (DINAEL), Genoa University, 16126 Genoa, Italy \\ ${ }^{2}$ Department of Mechanical Engineering (DIME), Genoa University, 16126 Genoa, Italy
}

Correspondence should be addressed to Michele Viviani, viviani@dinav.unige.it

Received 2 February 2012; Accepted 10 May 2012

Academic Editor: Francesco Salvatore

Copyright ( 2012 Daniele Bertetta et al. This is an open access article distributed under the Creative Commons Attribution License, which permits unrestricted use, distribution, and reproduction in any medium, provided the original work is properly cited.

\begin{abstract}
In the present paper an experimental and numerical analysis of an unconventional CLT propeller is carried out. Two different numerical approaches, a potential panel method and an RANSE solver, are employed. Cavitation tunnel experiments are carried out in order to measure, as usual, thrust, torque, and cavity extension for different propeller working points. Moreover, LDV measurements are performed to have a deep insight into the complex wake behind the propeller and to analyze the dynamics of generated tip vortexes. The numerical/experimental analysis and comparison of results highlight the peculiarities of this kind of propellers, the possibility to increase efficiency and reduce cavitation risk, in order to exploit the design approaches already well proven for conventional propellers also in the case of these unconventional geometries.
\end{abstract}

\section{Introduction}

Energy saving is a primary objective, historically the first, and, probably still now, the most important one, in the design of marine propellers. The constant increase of oil price, the more strict regulations in terms of air pollution, and the limits for $\mathrm{NO}_{X}$ and $\mathrm{SO}_{X}$ emissions require more and more efficient designs. Modern design approaches, like fully numerical lifting line/lifting surface codes and optimization applied to potential panel methods satisfy this objective and allow to design conventional propellers with maximum efficiency for a given operating point. On the other hand, nonconventional propellers, like Contracted and Loaded Tip CLT and Kappel like geometries, represent a further opportunity to increase efficiency and reduce the risk of cavitation, without the employment of completely different propulsive solutions, like contra- and corotating propellers or by the adoption of ducts, stators or wake regularizers.

At the same time, also requirements in terms of radiated noise and vibration emissions became more strict: avoidance of negative effects on marine life and reduction of the risk of hydroacoustic signature are the primary aims of new commercial and navy constructions. CLT propellers may represent a valid answer to these demands, even if the effects of some peculiar phenomena, such as the cavitating vortices at tip, need further investigation.

The first concept of tip-loaded propellers goes back to late seventies: Tip Vortex Free propellers were the first application of the Loaded Tip concept, that quickly evolved toward the CLT solution when also contraction of the fluid vein has been taken into account for the definition of the optimal geometry (http://www.sistemar.com/).

CLT propellers are characterized by a monotonic increase of pitch from blade root to tip, a finite chord at tip, moderate values of skew, and an endplate at the outermost radial edge of the blade towards the pressure side. Fullscale installations and observations, together with model scale measures and theoretical studies, as reported by SISTEMAR (http://www.sistemar.com/) identified, as the main advantages of CLT propellers, higher values of efficiency (thus lower fuel consumption, air polluting lower emissions), higher value of thrust per unit area (thus higher ship speed and lower optimum diameter), lower noise, and vibration levels with better margin for face cavitation and cavitation inception speed.

The gain in efficiency is obtained by the displacement of the maximum load towards the tip, that is made possible, without high noise and energy losses (typical of tip loaded 
conventional propellers) by the presence of the endplate. In fact, the outer radial sections of the blade contribute more efficiently to the generation of thrust: velocities are higher and, geometrically, local pitch angle is lower, that is, the local lift is more "aligned" with the axial propeller direction. A way to achieve high efficiency is, thus, to produce the most part of the required propeller thrust in this region of the blade. The additional span (not in the radial direction) provided by the endplate allows to locate the maximum load near the blade tip with a gradual and smooth reduction of the loading curve. In this way, it is possible to avoid the presence of a strong tip vortex and the higher values of induced velocities on the propeller plane, whose effect on the hydrodynamic pitch is fundamental in achieving high values of efficiency.

The presence of the endplate itself, that increases the pressure difference on the tip region and allows to adopt a finite chord at tip, produces higher value of thrust per unit area and a local unload of the sections. The resulting smaller optimum diameter allows the propeller to operate in a more uniform hull wake, with wider propeller sections passing through areas where the change in local wake can be strong, achieving more stable cavity bubbles and less cavity induced vibrations (but higher fluctuations of delivered thrust).

However, several problems, connected with the peculiarities of this kind of propellers, still need to be investigated. The shape and position of the endplate poses some issues regarding local strength, the overall influence on propeller mechanical characteristics and the higher risk of "double" tip vortex cavitation. Tip vortexes are, in fact, the main sources of energy loss, propagated noise, and induced vibrations. Also, optimal unconventional propellers, of course, like conventional ones, have to deal with the interactions between incoming flow and generated vortical structures to achieve high efficiency. If the hydroacoustic noise and the fluctuating pressures on the hull have to be minimized (or, at least, monitored), as today more frequently requested directly during the preliminary propeller design, the knowledge of the tip vortexes dynamics becomes a key aspect for the noise characterization of these kind of unconventional propellers, with respect of which few literature is available for designers. The peculiarity of the propeller geometry, moreover, is expected to produce very complex wake fields: blade trailing wake is subjected to large deformations as a combination between skew distribution and interaction between tip vortexes at the endplate. Strength and position of vortex cores, that in general is a matter of interest for conventional propellers and, during the design phase, a key aspect for the accurate evaluation of load distribution (see, e.g., the wake alignment issues in [1-3]), requires further analyses, for which extensive experimental campaigns and accurate viscous computations are expected to provide valuable results. Scale effects, in addition, represent another challenging task for this kind of unconventional propellers, for which a deeper investigation is needed [4].

From a numerical point of view, a lot of interest has been dedicated to the analysis and development of new propulsion concepts [5] involving CLT propellers. The TRIPOD European Project, for instance, is an example of the application of numerical tools, like potential-flow based methods and RANSE codes, in order to reduce the number of propeller design iterations for new propulsive solutions, in which unconventional Contracted and Loaded Tip propellers are adopted in contrarotating and POD configurations. More recently, panel and RANSE solver have been adopted for the analysis of open water performances of CLT propellers, with satisfactory preliminary results [6].

In the present work, both the numerical approaches, a potential panel method and a RANSE code, are applied for the analysis of open water CLT propeller performances, including prediction of steady cavitation extent and propeller wake. While the former approach can be considered the best compromise between accuracy and computational time in the initial design stage (suitable to have an initial estimation of forces and cavitation extent), the latter represents a reliable tool to analyze the effects of viscosity that, especially in off-design conditions, where potential approaches generally fails, are the leading aspects. The reliability of both the approaches are investigated and their application limits for unconventional geometries highlighted through a dedicated experimental campaign carried out at the Cavitation Tunnel of the University of Genoa and comparing with previous results obtained during tests carried out at CEHIPAR towing tank. In particular, after some preliminary tests in order to evaluate mechanical characteristics to be compared with CEHIPAR results, LDV measurements in steady conditions have been conducted. Measurements include velocity/vorticity fields characterization on a series of transversal planes at different distances from the propeller disc, for a given operating condition. Results are compared with the numerical computations and the location and evolution of the tip vortexes are highlighted. Finally, cavity extension, are investigated at different load and cavitation index conditions. Also, in this case, comparison with computations provides useful information about the reliability of the numerical methodologies adopted.

The experimental facilities are described in Section 2; Section 3 summarizes the theoretical aspects of the numerical approaches employed for the computations while in Section 4 the convergence of the numerical codes is checked. Section 5 presents the results (measured and computed), with an extensive comparison between numerical and experimental results and the evaluation of the main aspects of the phenomena under investigation.

\section{Experimental Setup}

Experiments were carried out at Cavitation Tunnel facility of the Department of Naval Architecture and Electrical Engineering of the University of Genoa (DINAEL), whose layout is schematically represented in Figure 1. The facility is a Kempf and Remmers closed water circuit tunnel with a squared testing section of $0.57 \mathrm{~m} \times 0.57 \mathrm{~m}$, having a total length of $2 \mathrm{~m}$. The nozzle contraction ratio is $4.6: 1$, and the maximum flow speed in the testing section is $8.5 \mathrm{~m} / \mathrm{s}$. Vertical distance between horizontal ducts is $4.54 \mathrm{~m}$, while horizontal distance between vertical ducts is $8.15 \mathrm{~m}$. Flow speed in the testing section is measured by means of a differential venturimeter with two pressure plugs immediately upstream 


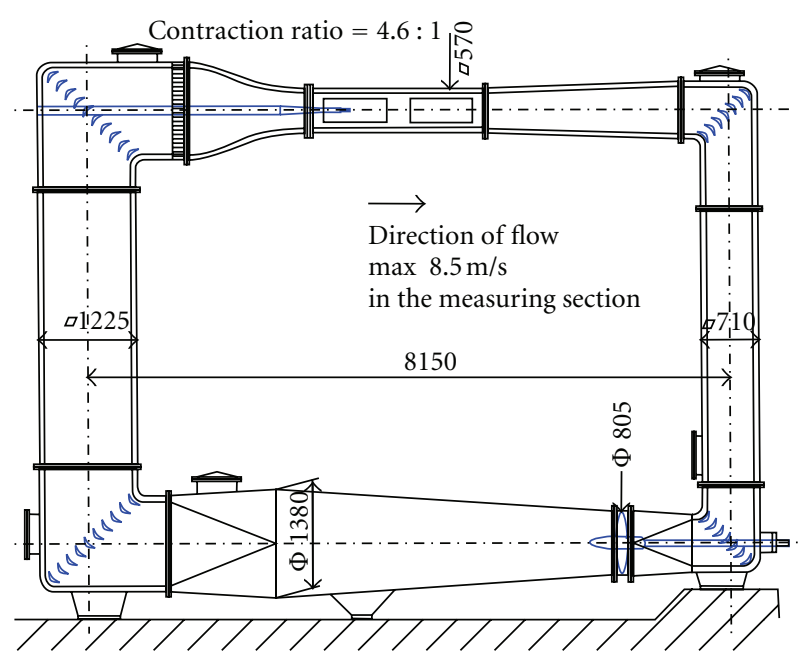

FIgURE 1: Main dimension of DINAEL cavitation tunnel.

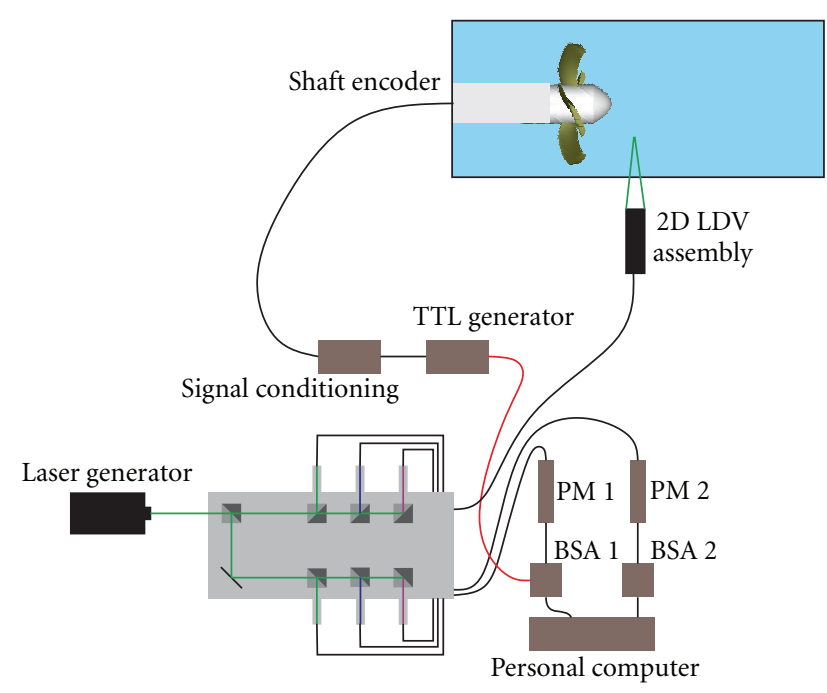

Figure 2: LDV system.

and downstream of the converging part. A depressurization system allows to obtain an atmospheric pressure in the circuit near to vacuum, in order to simulate the correct cavitation index for propellers and profiles (2D and 3D). The tunnel is equipped with a Kempf and Remmers H39 dynamometer, which measures propeller thrust, torque and RPM. As usual, a mobile stroboscopic system allows to visualize cavitation phenomena on the propeller blades. Moreover, cavitation phenomena visualization in the testing section is also made with a certain number (usually two/three) of cameras. Optical access to the testing section is possible through large windows: two Allied Vision Tech Marlin F145B2 Firewire Cameras, with a resolution of $1392 \times 1040$ pixels and a frame rate up to $10 \mathrm{fps}$ were adopted.

The Cavitation Tunnel is also equipped with instrumentation for nonintrusive measurement of velocity field, that is, Laser Doppler Velocimeter (Figure 2).

Measurements of the three velocity components of the flow upstream and downstream of the propeller have been performed by means of a four-beam two-color fibre optic
LDV system with back-scatter collection optics (Dantec Fiber Flow). The light source is a $5 \mathrm{~W}$ argon ion laser operating at $514.5 \mathrm{~nm}$ (green), $488 \mathrm{~nm}$ (blue). In order to solve the zero velocity ambiguity and to reduce angle bias, a $40 \mathrm{MHz}$ Bragg cell has been used. The probe consists of an optical transducer head of $60 \mathrm{~mm}$ diameter, with a focal length of $400 \mathrm{~mm}$ and a beam separation of $38 \mathrm{~mm}$, connected to the emitting optics and to the photomultipliers by means of optic fibres. Typical probe volume dimensions were $190 \mu \mathrm{m}$ diameter and $4 \mathrm{~mm}$ length. The signal from the photomultipliers has been processed by two Dantec Burst Spectrum Analysers. Probe assembly is stiffly mounted on a threeaxis computer-controlled probe traversing mechanism. The motion is transmitted to the carriages by stepping motors through a preloaded ball-screw assembly with a minimum linear translation step of $8 \mu \mathrm{m}$ [7].

In the present experiment, an ensemble averaging technique suitable for LDV data processing has been applied. A reference signal has been derived directly from the propeller shaft encoder, and hence can be considered in phase with the relevant periodic phenomenon. To obtain statistically accurate ensemble averages, a total of 100000 validated data for each velocity component have been sampled at each measuring position. Instantaneous velocities are sorted into 360 phase bins, each representing a particular phase of the cycle. Taking into account the direct relation between period of the cycle and propeller rotational speed, each bin represents a particular angular position among a total of 360 .

A comprehensive review of errors in laser-Doppler velocimetry measurements and guidelines to evaluate them is given in Min [8], Lakshminarayana [9], Boutier [10], Strazisar [11] and Modarress et al. [12]. Statistical uncertainty in mean and rms velocities depends on the number of sampled data, turbulence intensity, and confidence level. For the present experiment, considering a confidence level of 95 per cent, a local turbulence intensity of 20 per cent and a minimum number of samples of 150 for bin, uncertainties of \pm 3 and \pm 7 per cent are expected for the mean and rms velocities, respectively.

A generic instantaneous quantity (i.e., velocity component) is a function of time $\left(t_{j}\right)$ or rotor circumferential coordinate, of the data record $(n)$.

The equations defining the ensemble average procedure are as follows:

(i) instantaneous quantity:

$$
q\left(t_{i}, n\right)=\tilde{q}\left(t_{i}\right)+q^{\prime}\left(t_{i}, n\right)
$$

(ii) ensemble averaged quantity:

$$
\tilde{q}\left(t_{j}\right)=\frac{1}{N} \sum_{n=1}^{N} q\left(t_{j}, n\right)
$$

(iii) root mean square of the random unsteady fluctuations:

$$
r \tilde{m} s(q)=\sqrt{\widetilde{q^{\prime 2}}\left(t_{j}\right)}=\sqrt{\frac{1}{N-1} \sum_{n=1}^{N}\left[q\left(t_{j}, n\right)-\tilde{q}\left(t_{j}\right)\right]^{2}} .
$$




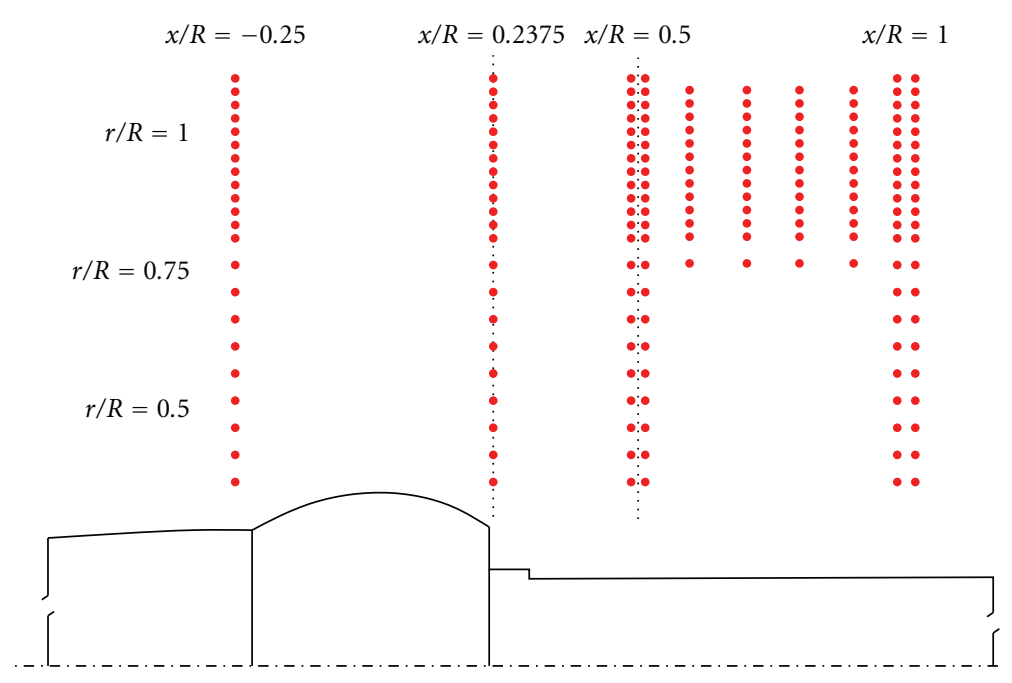

Figure 3: Effective measurement points.

TABLE 1: Measurements stations.

\begin{tabular}{|c|c|c|c|c|c|c|c|c|c|c|}
\hline & \multicolumn{10}{|c|}{ Station } \\
\hline & 1 & 2 & 3 & 4 & 5 & 6 & 7 & 8 & 9 & 10 \\
\hline Axial position $(x / R)$ & -0.25 & 0.2375 & 0.4875 & 0.5125 & 0.6 & 0.7 & 0.8 & 0.9 & 0.9875 & 1.0125 \\
\hline
\end{tabular}

In order to survey all the three velocity components using a 2D LDV it has been necessary to perform measurements in two different configurations: the first one through the upper window of the cavitation tunnel, the second one through a lateral window. Special attention has been dedicated to the selection of the most appropriate measurement configuration in order to maximize spatial measurement resolution and limit the smoothing of velocity gradient.

Measurements have been performed for ten axial stations, described in details in Table 1. Effective measured points (along the radial direction) are highlighted in Figure 3: for the first four stations and for the last two, measures cover the complete radial extension (from $r / R=$ 0.35 to $r / R=1.1$ ) of the propeller wake, while for the stations between $x / R=0.6$ and $x / R=0.9$, only points in the tip region have been investigated, because the main interest of the measurements was to analyze the evolution of tip vortices. Stations $3 / 4$ and 9/10 have been measured at small axial distance, in order to evaluate vorticity at the intermediate section.

The reference coordinate system adopted for the presentation of results and the conventions on the sign of axial, radial and tangential velocities are summarized in Figure 4.

\section{Numerical Methods}

The numerical modeling of Contracted and Tip Loaded Propellers, from and hydrodynamic point of view is equivalent to the conventional propellers case. Once the geometry has been defined, paying special attention to the modeling of the endplate, the hydrodynamic characteristics of CLT propellers can be computed straightforward applying both potential and RANSE solvers.

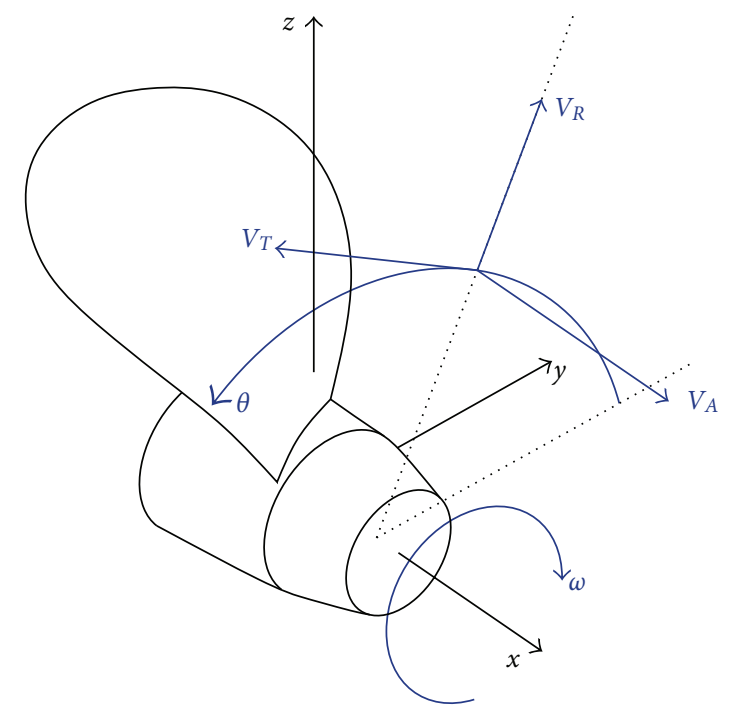

FIGURE 4: Coordinate reference system and velocity convention.

3.1. Panel Method. Panel/boundary elements methods model the flowfield around a solid body by means of a scalar function, the perturbation potential $\phi(\mathbf{x})$, whose spatial derivatives represent the component of the perturbation velocity vector. Irrotationality, incompressibility, an absence of viscosity are the hypothesis needed in order to write the more general continuity and momentum equations as a Laplace equation for the perturbation potential itself:

$$
\nabla^{2} \phi(\mathbf{x})=0
$$


For the more general problem of cavitating flow, Green's third identity allows to solve the three dimensional differential problem as a simpler integral problem written for the surfaces that bound the domain. The solution is found as the intensity of a series of mathematical singularities (sources and dipoles) whose superposition models the inviscid cavitating flow on and around the body:

$$
\begin{aligned}
2 \pi \phi\left(\mathbf{x}_{p}\right)= & \int_{S_{B}+S_{\mathrm{CB}}} \phi\left(\mathbf{x}_{q}\right) \frac{\partial}{\partial \mathbf{n}_{q}} \frac{1}{\mathbf{r}_{p q}} d S-\int_{S_{B}+S_{\mathrm{CB}}} \frac{\partial \phi\left(\mathbf{x}_{q}\right)}{\partial \mathbf{n}_{q}} \frac{1}{\mathbf{r}_{p q}} d S \\
& +\int_{S_{W}} \Delta \phi\left(\mathbf{x}_{q}\right) \frac{\partial}{\partial \mathbf{n}_{q}} \frac{1}{\mathbf{r}_{p q}} d S .
\end{aligned}
$$

Neglecting the supercavitating case (computation is stopped when the cavity bubble reaches the blade trailing edge) and assuming that the cavity bubble thickness is small with respect to the profile chord, [13] singularities that model cavity bubble can be placed on the blade surface other than on the real cavity surface, leading to an integral equation in which the subscript $q$ corresponds to the variable point in the integration, $\mathbf{n}$ is the unit normal to the boundary surfaces, and $\mathbf{r}_{p q}$ is the distance between points $p$ and $q, S_{B}$ is the fully wetted surface, $S_{W}$ is the wake surface, and $S_{\mathrm{CB}}$ is the projected cavitating surface on the solid boundaries. This approach can be considered as a partial nonlinear approach that takes into account the weakly nonlinearity of the boundary conditions (the dynamic boundary condition on the cavitating part of the blade and the closure condition at its trailing edge) without the need to collocate the singularities on the effective cavity surface. The set of required boundary conditions for the steady problem is as follow.

(i) Kinematic boundary condition on the wetted solid boundaries:

$$
\frac{\partial \phi(\mathbf{x})}{\partial \mathbf{n}}=-\mathbf{V}_{\text {inflow }}(\mathbf{x}) \cdot \mathbf{n} .
$$

(ii) Kutta condition at blade trailing edge (iteratively solved as a stagnation pressure condition):

$$
\nabla|\phi|_{\text {T.E. }}<\infty \text {. }
$$

(iii) Dynamic boundary condition on the cavitating surfaces:

$$
p=p_{\text {vap }} \quad \text { on } S_{\mathrm{CB}} \text {. }
$$

(iv) Kinematic boundary condition on the sheet cavity surface, where $\mathbf{n}$ is the surface normal vector and th is the local cavity bubble thickness evaluated on a $\mathbf{l}, \mathbf{m}, \mathbf{n}$ local nonorthogonal reference system:

$$
\frac{D}{D t}(\mathbf{n}-t h(\mathbf{l}, \mathbf{m}))=0 .
$$

(v) Cavity closure condition at cavity bubble trailing edge.
Arbitrary detachment line, on the back and/or on the face sides of the blade can be found, iteratively, applying a criteria equivalent, in two dimensions, to the Villat-Brillouin cavity detachment lines, as in Mueller and Kinnas [14]. Starting from a detachment line obtained from the initial wetted solution (and identified as the line that separates zones with pressures higher than the vapor tension from zones subjected to pressure equal or lower pressures) or an imposed one (typically the leading edge), the detachment line is iteratively moved according to the following.

(i) If the cavity at that position has negative thickness, the detachment location is moved toward the trailing edge of the blade.

(ii) If the pressure at a position upstream the actual detachment line is below vapor pressure, then the detachment location is moved toward the leading edge of the blade.

The numerical solution consists in an iterative scheme delegated to solve the nonlinearities connected with the Kutta, the dynamic, and the kinematic boundary conditions on the unknown cavity surfaces until the cavity closure condition has been satisfied.

Viscous forces, neglected by the potential flow method, can be added in general with two different approaches. In the first case, as proposed by Hufford [15] and Gaggero [2], a thin boundary layer solver can be coupled, through transpiration velocities, to the inviscid solution, in order to obtain a local estimation of the frictional coefficient computed in accordance to the integral approach of Curle [16] (for the laminar boundary layer) and Nash and Hicks [17] (for the turbulent boundary layer). This approach, though being applied successfully for the analysis of conventional propellers, poses some problems of convergence in very off-design conditions and suffers from the tip influence on streamlines on which the boundary layer calculation is performed. As a consequence in the present work, a local estimation of frictional coefficient has been carried out applying a standard frictional line. In particular, a formulation, based on local Reynolds number and thickness/chord ratio, has been employed:

$$
C_{D}(r)=\frac{1}{2} a\left(1+b \frac{t(r)}{c(r)}\right) \operatorname{Re}_{N}^{k}(r) .
$$

3.2. RANSE Solver. Viscous analysis of open water and cavitating propeller characteristics has been carried out through StarCCM+, a commercial finite volume RANSE solver [18]. The hypothesis of incompressible fluid, if also vapor due to cavitation is taken into account, holds only in an approximate way. However, if the acoustic problem is not under investigation, the assumption of incompressible flow lets to solve a more computationally efficient numerical model, in which both the phases, water and vapor, are incompressible and interact only through the continuity equation. Only one "mixed" fluid needs to be solved, which proprieties are a weighted mean between the fraction $(\alpha)$ of liquid $(l)$ and the fraction $(\alpha-1)$ of vapor $(v)$ characteristics: 


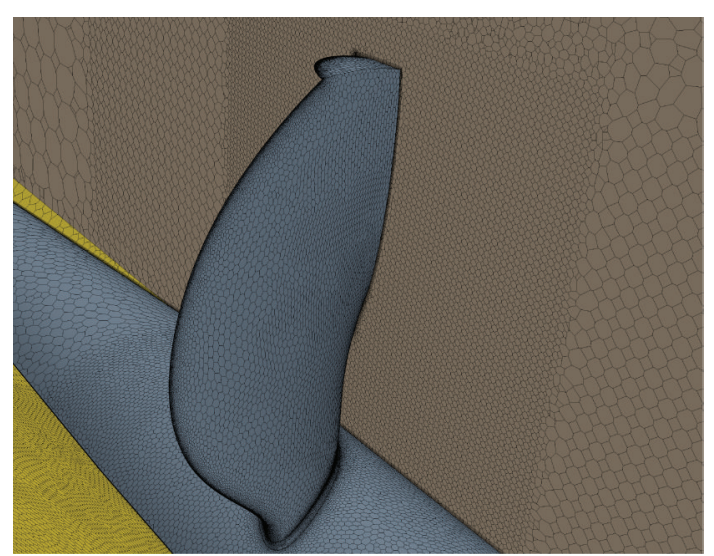

Figure 5: Mesh refinements close to the blade. Finer mesh (1.6 million cells).

$$
\begin{aligned}
& \rho_{\text {mix }}=\rho_{l}(\alpha)+\rho_{v}(1-\alpha), \\
& \mu_{\text {mix }}=\mu_{l}(\alpha)+\mu_{v}(1-\alpha) .
\end{aligned}
$$

The governing equations (continuity, momentum and an additional transport equation for the fraction $\alpha$ ) for the mixture can be written as:

$$
\begin{gathered}
\nabla \cdot \mathbf{U}=0 \\
\rho_{\text {mix }} \dot{\mathbf{U}}=-\nabla p+\mu_{\text {mix }} \nabla^{2} \mathbf{U}+\nabla \cdot \mathbf{T}_{\mathrm{Re}}+\mathbf{S}_{M} \\
\frac{\partial \alpha}{\partial t}+\nabla \cdot(\alpha \mathbf{U})=\frac{\dot{m}_{c}+\dot{m}_{r}}{\rho_{l}}
\end{gathered}
$$

in which $\mathbf{U}$ is the averaged velocity vector, $p$ is the averaged pressure field, $\mu_{\text {mix }}$ and $\rho_{\text {mix }}$ are the mixed dynamic viscosity and the mixed density, $\mathbf{S}_{M}$ is the momentum sources vector, $\mathbf{T}_{\mathrm{Re}}$ is the tensor of Reynolds stresses computed according to the Realizable $k-\varepsilon$ turbulence closure equations, and $\left(\dot{m}_{c}+\right.$ $\left.\dot{m}_{r}\right) / \rho_{l}$ is the cavitation source term.

The StarCCM+ computational domain, by means of the symmetries, is represented by an angular sector of amplitude $2 \pi / Z$ around a single blade, discretized with an appropriate unstructured mesh of polyhedral cells chosen at the light of the convergence analysis carried for all the addressed problem (Figure 5). Simulations for the computation of open water propeller characteristics and propeller wake have been carried out as steady, using the Moving Reference Frame approach, whit SIMPLE algorithm to link pressure with velocity fields. Cavitation has been evaluated, instead, with an implicit unsteady approach, with a fixed time step of $5 \cdot 10^{-5}$ seconds.

\section{Convergence of the Numerical Approaches}

Before proceeding with the analysis of the computed results through the comparison with the experimental measures, a preliminary analysis of the convergence of the numerical solution for both the selected solvers has been carried out. Open water performances, propeller wake, and cavity extension, the main aspects of interest for which the numerical and experimental investigations have been performed, have been considered for the evaluation of the convergence behavior of the numerical solutions. In open water and in cavitating condition, both the approaches have been investigated. Instead, convergence of the propeller wake has been verified only for the RANSE solver (the panel method, in fact, has not been employed for these kind of computations).

Figure 6 shows open water thrust and torque coefficients as a function of the number of panels on the blade surface (panel method) and of the number of cells on the fluid domain (RANSE). Computations have been carried out at an advance coefficient equal to 0.8 at $18 \mathrm{~Hz}$, for which a thrust coefficient of 0.1839 (towing tank) and a torque coefficient $\left(10 K_{Q}\right)$ of 0.3659 (towing tank) are expected. Number of panels for each blade plus its relative hub varies from about 900 (15 radial sections and 30 points along the profile) to about 2400 (30 sections and 60 points along the profile), with a trailing vortical wake, discretized with an equivalent angular spacing of 6 degree, extended for six complete revolutions aft the trailing edge. Calculations were carried out with a fixed wake model in which the pitch is the mean between the geometrical propeller pitch and the hydrodynamic pitch, since this approach already proved to be reliable and robust, as shown in Gaggero, [2]. The maximum number of cells (1.6 million/blade) for the viscous computations has been achieved through successive refinement of the initial mesh (460 thousands elements/blade) close to the propeller, as shown in Figure 5. In any case, an appropriate prism layer (five layer with growing factor of 1.25), fitted on the wall boundaries, has been realized with a thickness evaluated from empirical boundary layer formulations.

An overall good convergence has been achieved for both the solvers, but with opposite tendencies. Predicted thrust and torque by panel method, in fact, tends to increase with the number of panels (extrapolated values are 0.1859 and 0.3768 resp., for the thrust and the torque coefficient) while RANSE computations have a decreasing behavior, with extrapolated values of 0.1764 , and 0.3551 respectively.

The computations of open water performances, as reported in next section, have been finally performed with the medium grid $(25 \times 50$ for the panel method, 1 Million cell for the RANSE) as a compromise between accuracy (with respect to the finer grid differences are, in both cases, lower than 1\%) and available computational resources. A similar analysis is required when the evaluation of propeller wake is addressed. In this case a finer mesh is mandatory in order to capture the peculiar vortex structures at the blade tip produced by the endplate and to avoid the numerical dissipation that tends to smooth the flow quantities, velocity, and vorticity distributions first of all.

The convergence of the numerical solution, also for this kind of computation, has been verified on three different meshes, obtained by refining the tip region where the higher gradients are expected. Figure 7 shows the base mesh (Figure $7(\mathrm{a})$ ), of about 1.5 Millions cells refined close to 


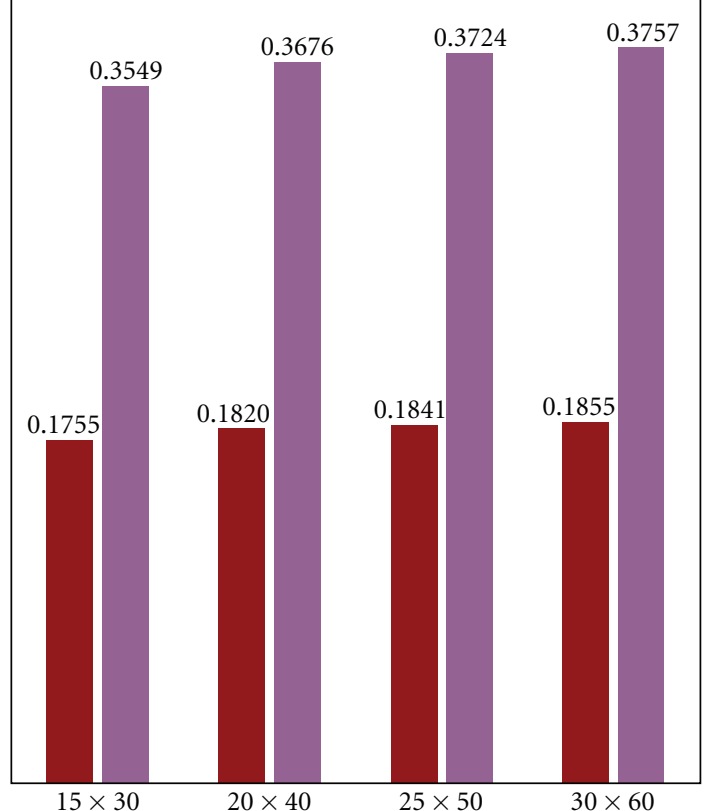

- $K_{T}$ panel

- $10 K_{Q}$ panel

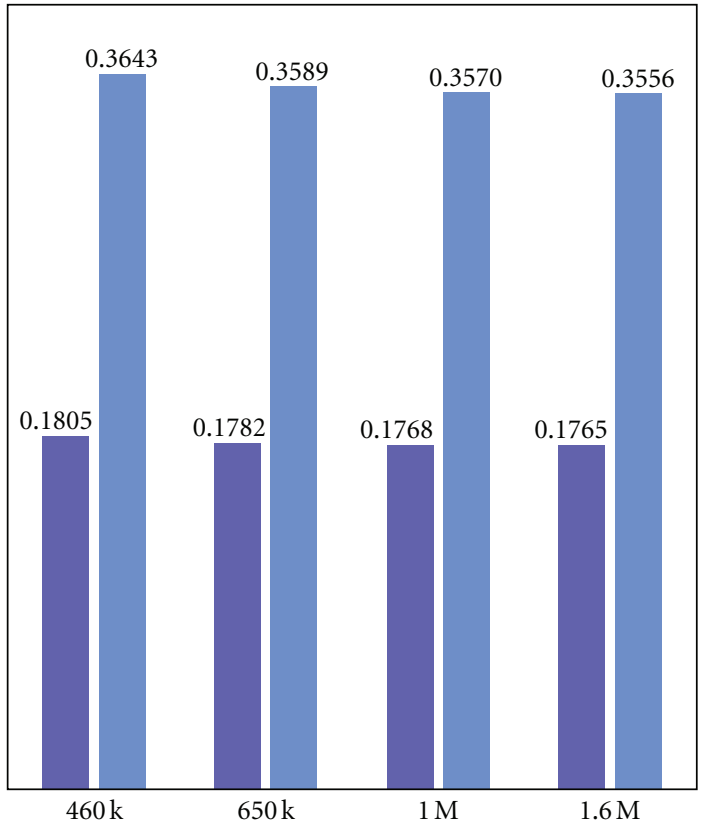

- $K_{T}$ RANSE

- $10 K_{Q}$ RANSE

(a)

(b)

Figure 6: Convergence of open water thrust and torque coefficient with the number of panels and the number of cells for the potential and the RANSE solution.

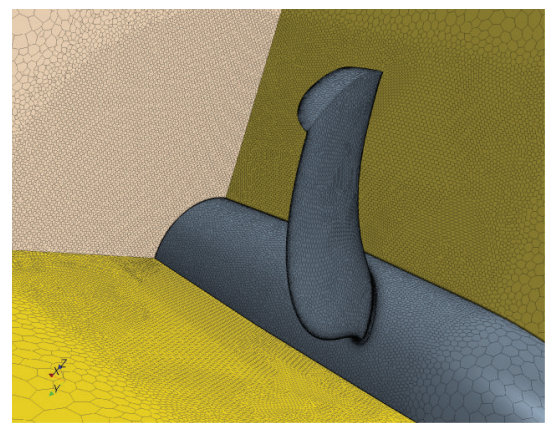

(a)

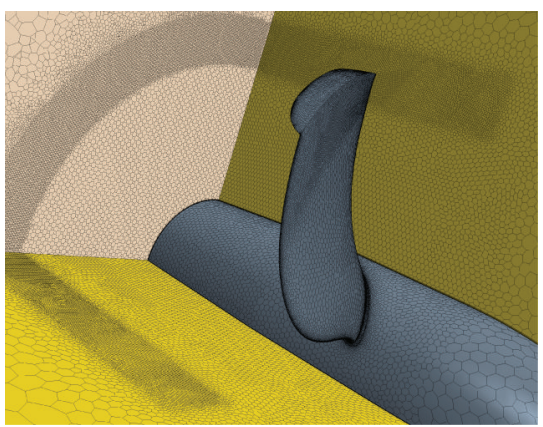

(b)

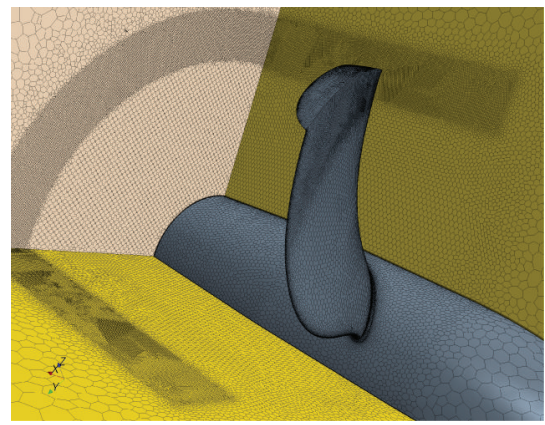

(c)

Figure 7: Mesh arrangements for propeller wake analysis. (a) Base mesh. (b) Medium mesh. (c) Finer mesh.

the blade, and the medium (Figure 7(b)) and the very fine (Figure 7(c)) meshes, respectively, of about 3.5 and 8 Millions cells, with a higher cell density in the tip region. An overview of the results is presented in Figure 8, in which nondimensional tangential and radial velocity distributions, computed on a section placed $0.56 R$ aft the propeller plane. More in detail, Figure 9 compares tangential and radial velocity on the same plane but for a section at constant radius equal to $0.9 R$.

Qualitatively the convergence of the solution is achieved directly from the comparison of the velocity field aft the propeller. If with the coarse mesh, it is almost impossible to identify the details of the double tip vortex structure experimentally observed (as in Figure 21, e.g., in which the tip vortexes are tracked by the cavitation phenomena) at the cavitation tunnel, the medium, and, definitely, the fine mesh grants a sufficient resolution to capture velocity peaks due to the interactions of the trailed vortical structures aft the propeller.

As better noted from Figure 9, the coarser mesh results in a considerable numerical smoothing of the velocity. Considering tangential velocity, it is possible, also with the coarser mesh, to identify the two velocity peaks (also if highly underpredicted with respect to the computations carried out by the medium and the fine resolution); regarding radial velocity distribution, it is clear that the coarse mesh is 


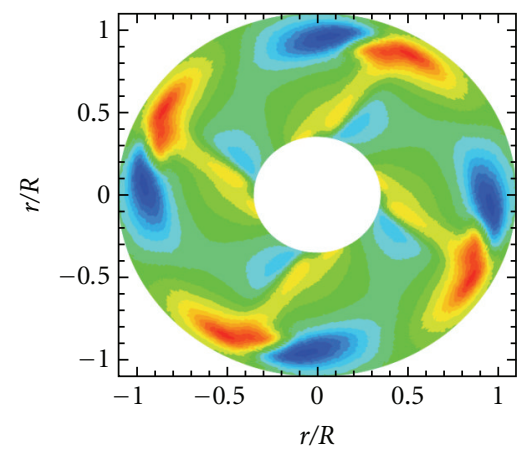

(a)

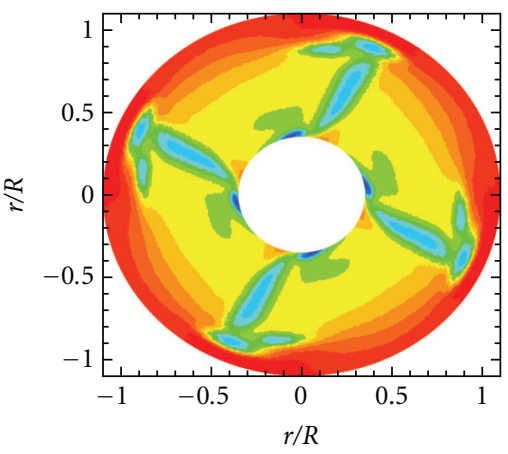

(d)

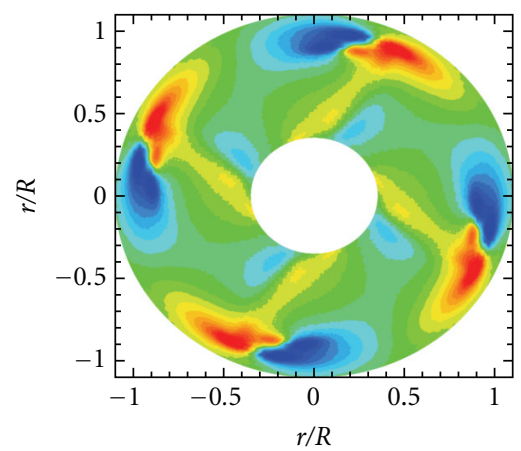

(b)

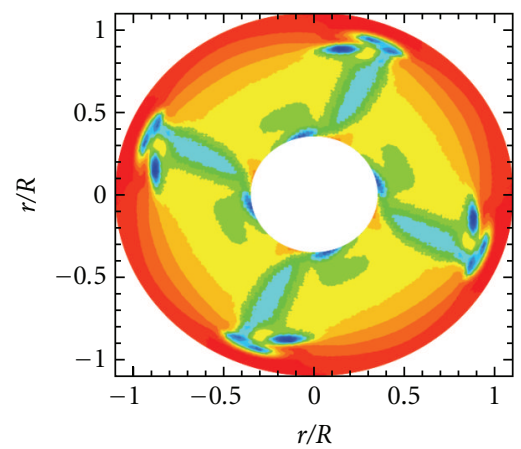

(e)

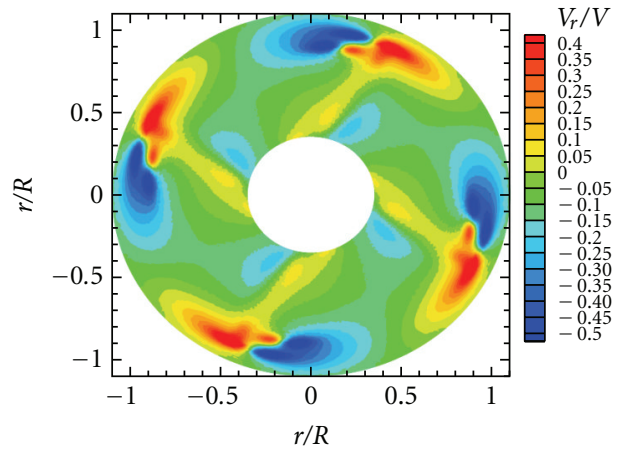

(c)

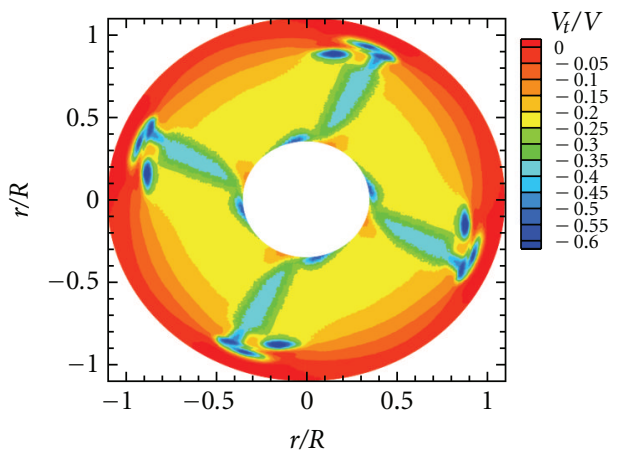

(f)

FIGURE 8: Convergence of radial and tangential nondimensional velocity distribution at $x / R=0.56$ aft the propeller plane. ((a) and (d)) Base mesh. ((b) and (e)) Medium mesh. ((c) and (f)) Finer mesh.

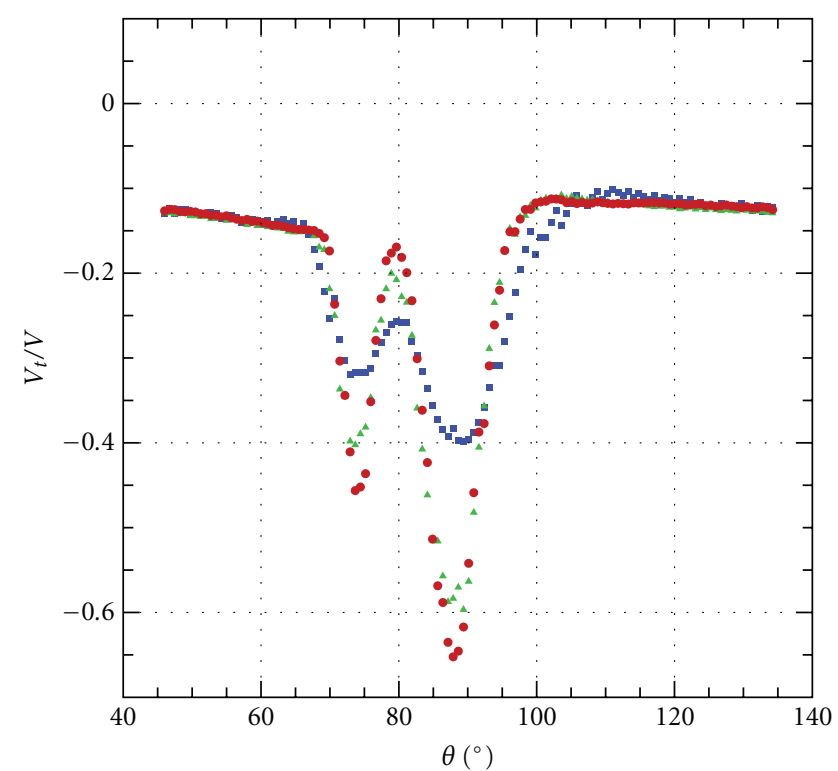

- $V_{t} / V$ coarse mesh

^ $V_{t} / V$ medium mesh

- $V_{t} / V$ fine mesh

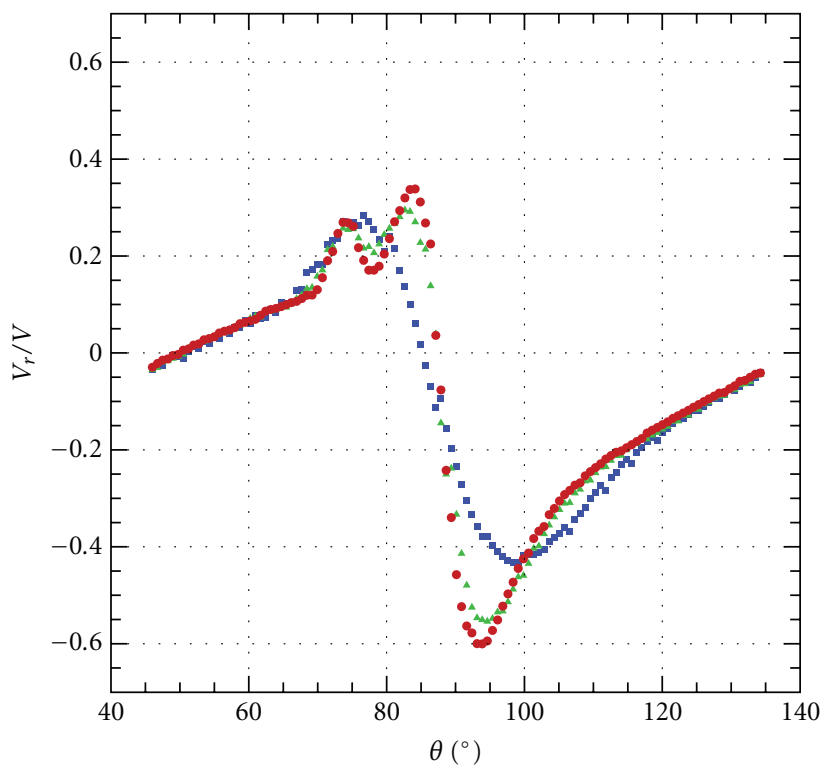

- $V_{r} / V$ coarse mesh

- $V_{r} / V$ medium mesh

- $V_{r} / V$ fine mesh

(a)

(b)

FIgURE 9: Convergence of radial and tangential nondimensional velocity distribution at $x / R=0.56, r / R=0.90$ aft the propeller plane. 


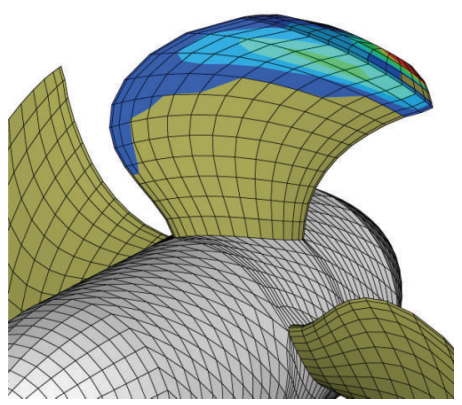

(a)

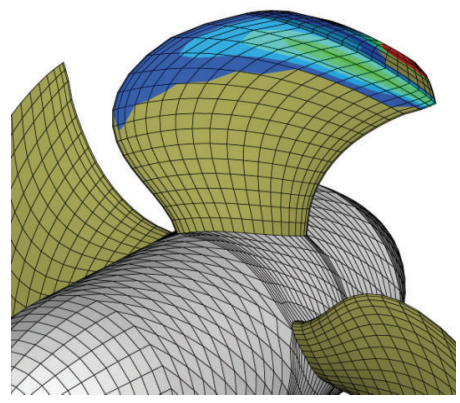

(b)

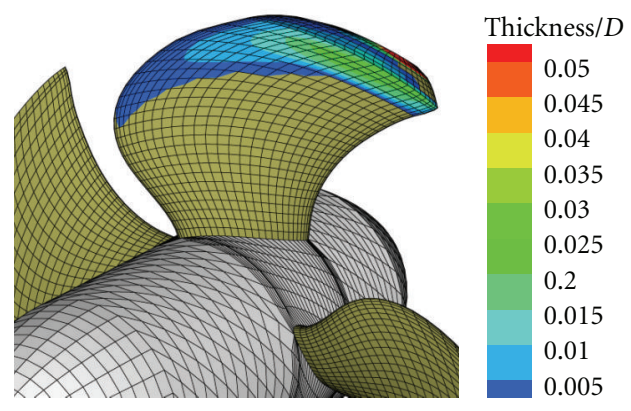

(c)

Figure 10: Convergence of cavity extension with the number of panels. Open water thrust coefficient equal to 0.213 at cavitation index of 1.625 .

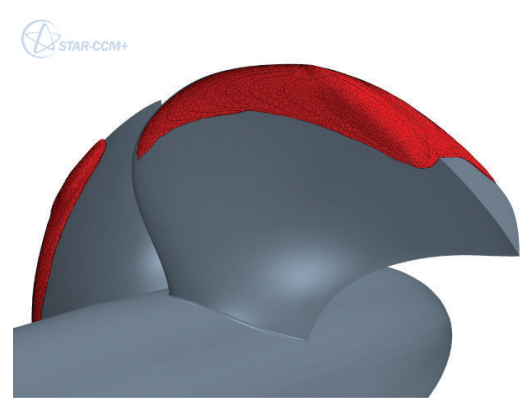

(a)

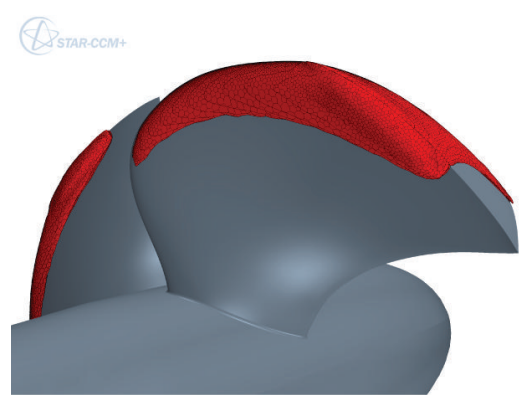

(b)

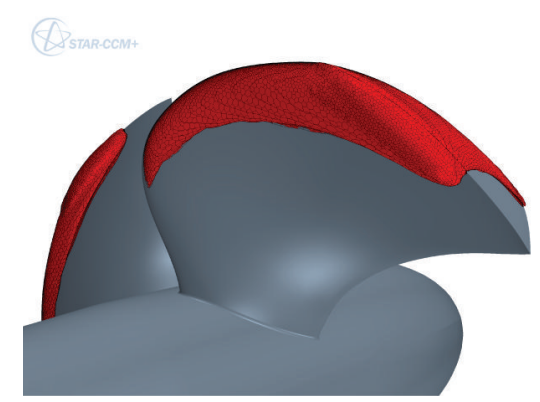

(c)

FIGURE 11: Convergence of cavity extension (fraction of vapor equal to 0.5 ) with the number of cells. Open water thrust coefficient equal to 0.213 at cavitation index of 1.625 .

inadequate to resolve the local flow behavior for which, instead, both the medium and the fine resolution captures the double peak and the shifting toward lower angles of the minimum values of the radial velocity.

The dependency of the solution on the discretization level, finally, has been verified in cavitating condition. Also, in this case, three different meshes, for the potential solver and for the viscous solution, have been investigated. As usual for the potential solution, the parameters under investigation (from which the whole discretization depends) are the number of radial sections (between 15 and 25) and the number of points along the chord (between 30 and 50), as evidenced in Figure 10. For the RANSE computations, instead, meshes have been realized changing the global base size, the number of prism layers, and the local minimum size of surface discretization on the blade. Resulting meshes have a number of cells between 500 thousands (coarse) and 1.5 Million (fine) for each blade, with discretization parameters summarized in Table 2.

Results, computed for a cavitation index equal to 1.625 and for an advance coefficient for which the corresponding open water thrust coefficient is equal to 0.213 , are compared, in terms of cavity extension, in Figure 10 (panel method, nondimensionalized cavity thickness with respect to the diameter) and in Figure 11 (RANSE, isosurface of volume fraction of vapor equal to 0.5$)$. The increased number of panels smoothes the potential solution, with a clear decrease

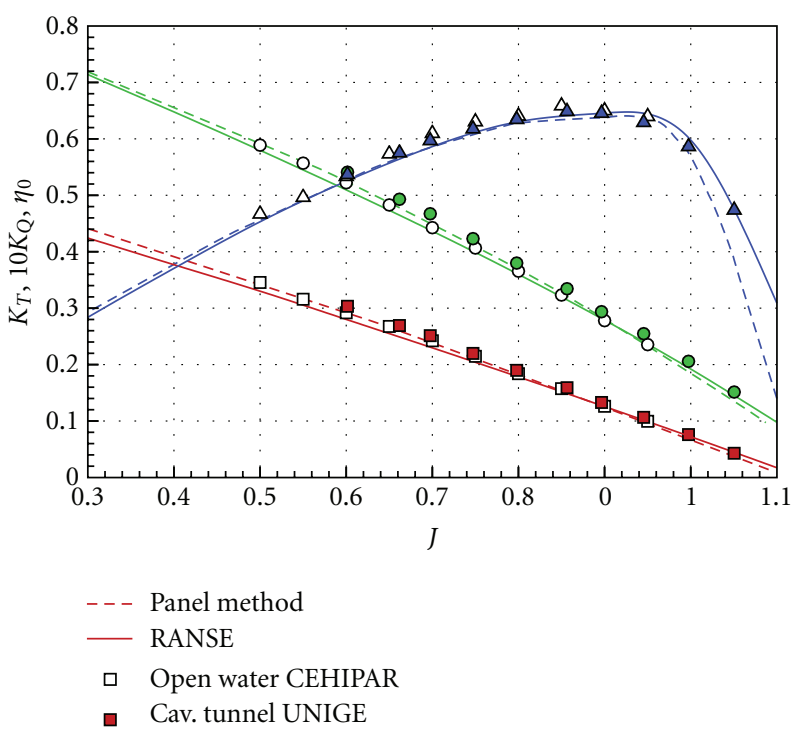

FIgURE 12: Open water propeller characteristics. RANSE and panel method in model scale compared with experimental towing tank/cavitation tunnel measures.

of the radial extension of the cavity bubble at the blade leading edge. Higher number of cells, instead, produces a slightly extended cavity region within the viscous approach. 
TABLE 2: Discretization parameters for RANSE meshes—cavitating case.

\begin{tabular}{lccc}
\hline & Coarse mesh & Medium mesh & Fine mesh \\
\hline Base size & 1.8 times prop. diam. & 1.8 times prop. diam. & 1.8 times prop. diam. \\
Prism layer thickness & $2 \mathrm{~mm}$ & $2 \mathrm{~mm}$ & $2 \mathrm{~mm}$ \\
Number of prism Layers & 8 & 10 & 15 \\
Minimum mesh surface size & $0.04 \%$ of base size & $0.02 \%$ of base size & $0.02 \%$ of base size \\
Target mesh surface size & $5 \%$ of base size & $2.5 \%$ of base size & $2.5 \%$ of base size \\
Total number of cells/blade & $\sim 500 \mathrm{k}$ & $\sim 1 \mathrm{M}$ & $\sim 1.5 \mathrm{M}$ \\
\hline
\end{tabular}

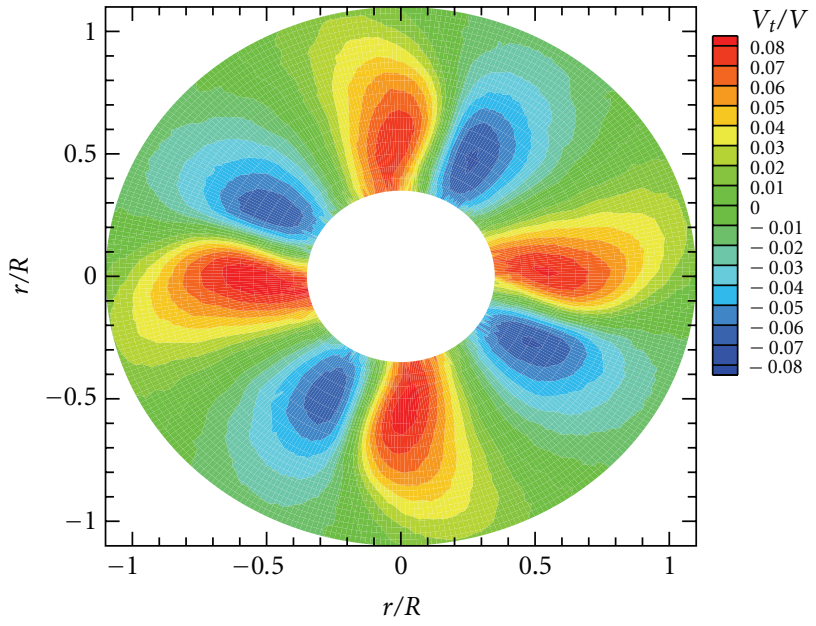

(a)

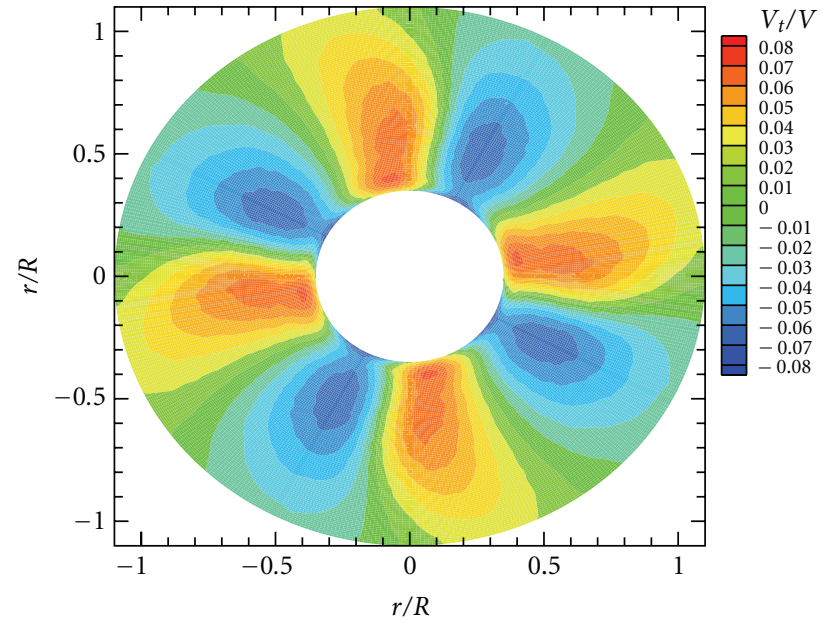

(b)

FIGURE 13: Tangential nondimensional velocity distribution at $x / R=0.2500$ fore the propeller plane. (a) Experimental LDV measurements. (b) RANSE computations on the finer mesh.

In particular, the influence of the discretization level is higher at the tip. Smaller cells reduce the numerical dissipation and a sharpen solution is obtained at endplate tip with an outline of cavitating tip vortex, for the computation of which a sensibly higher number of cells (comparable with that adopted for propeller wake evaluation) is required. The finer resolution for both the solvers, despite the higher computational effort required for the viscous computations, has been finally chosen for all the further calculations as a reliable level of discretization.

\section{Results}

As already discussed, CLT propellers are claimed to be unconventional propellers able to grant some advantages with respect to conventional solutions. In particular, especially at full scale, main advantages are higher efficiency, reduced cavitation, and tip vortex strengthand, in general, smaller propeller optimum diameter due to the high delivered thrust. The numerical analyses and the experimental measures, consequently, have been carried out having in mind these primary aspects: prediction/measure of propeller performances in open water, including the analysis of the propeller wake, and the prediction/measure of the steady cavity extension in design and off-design conditions. From the experimental point of view, the attention has been primarily focused on the characterization of the peculiar wake field downstream the propeller and of the steady cavity behavior as a function of load and cavitation index. Objectives of the analyses are the validation of the numerical codes both in non cavitating and in cavitating conditions and the evaluation of the capabilities of the panel method in predicting performances and the main features of the cavity bubble also for unconventional propellers.

5.1. Open Water. Model scale open water computations, compared with measures carried out at CEHIPAR towing tank [19] and at the University of Genoa Cavitation tunnel are reported in Figure 12. In all cases, tests have been carried out with $0^{\circ}$ shaft inclination and uniform flow (no wake) at $20 \mathrm{~Hz}$ in order to achieve a sufficiently high Reynolds number. Both the numerical predictions (Panel Method and RANSE solver) are very close to the experimental values for a wide range of advance coefficients. In mean, the difference between computed and measured values is less than $3 \%$ around the design point: panel method tends to overestimate both thrust and torque, while RANSE results are slightly underpredicted. Numerical results are provided in a wider range than the available experimental measures. These results show a common trend, with slightly higher values for $K_{T}$ and $K_{Q}$ at lower advance coefficients and a lower zero thrust point in case of panel method. Nevertheless, 


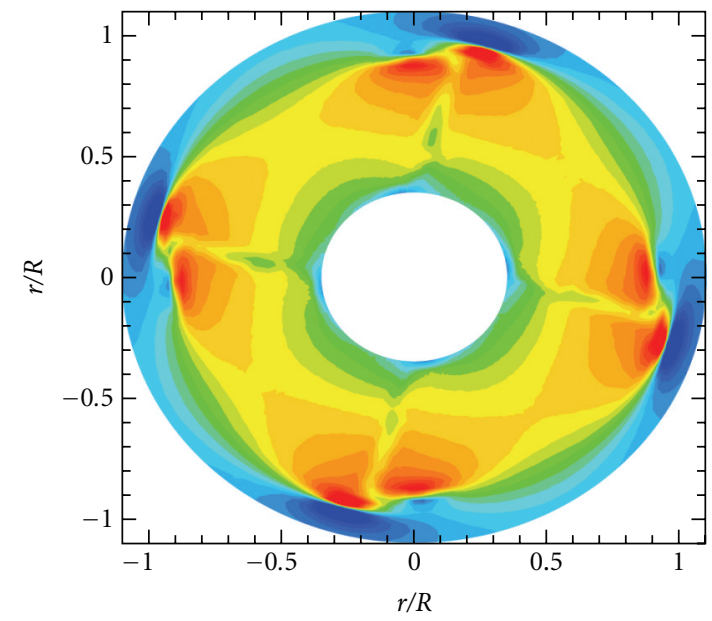

(a)

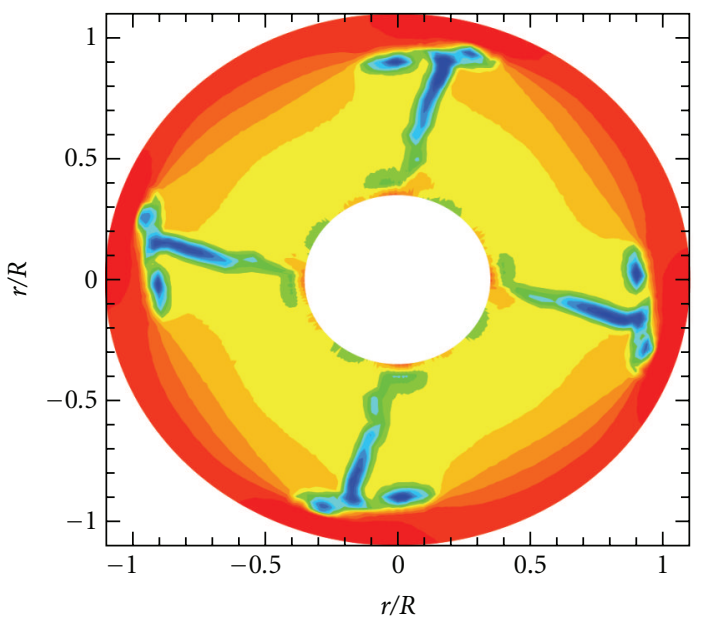

(c)

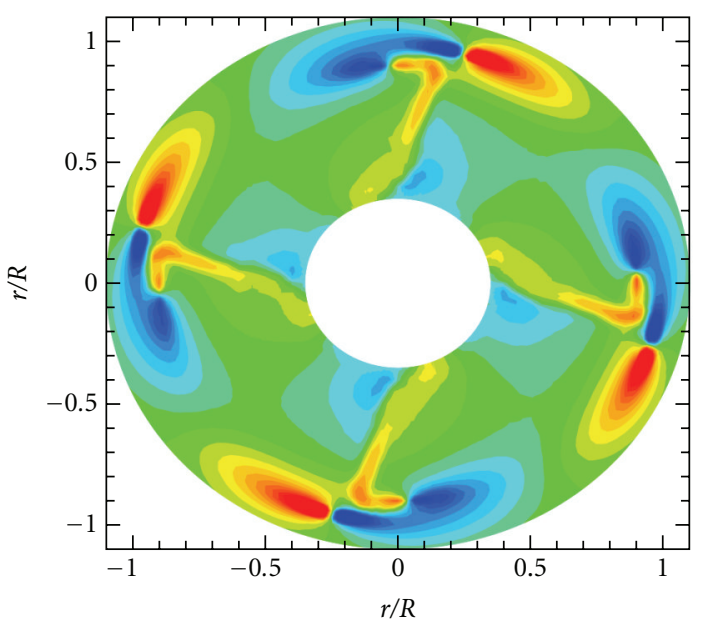

(e)
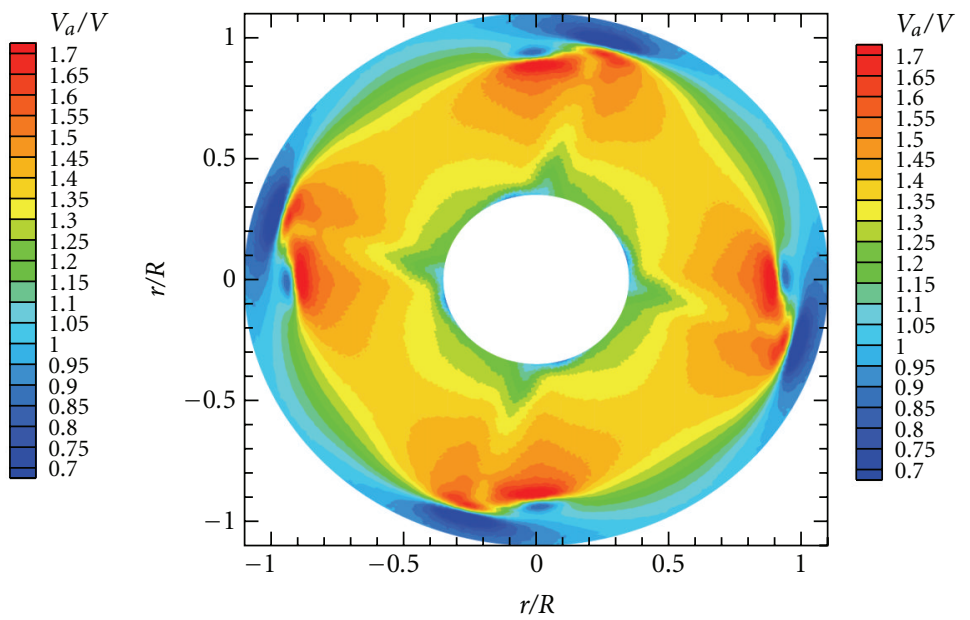

(b)

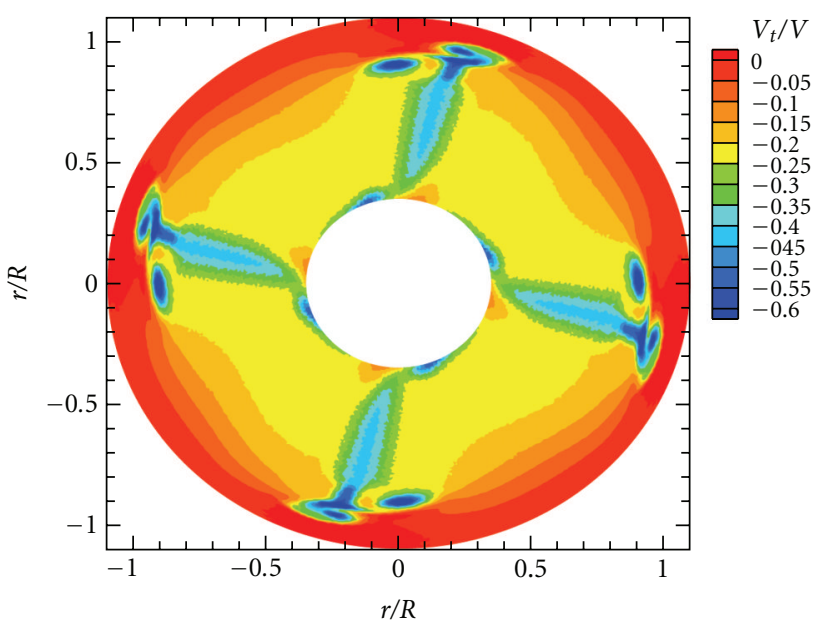

(d)

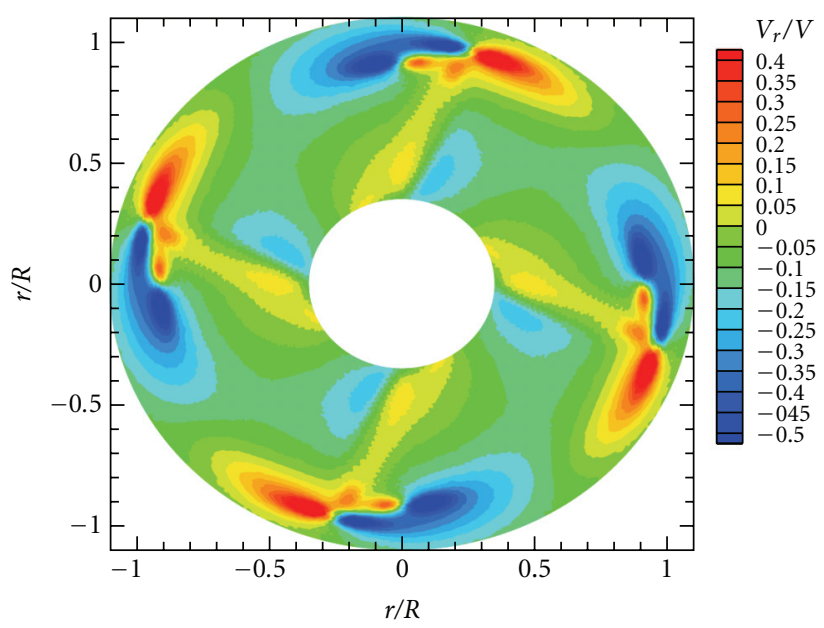

(f)

Figure 14: Axial, tangential, and radial nondimensional velocity distributions at $x / R=0.4875$ aft the propeller plane. ((a), (c), and (e)) Experimental LDV measurements. ((b), (d), and (f)) RANSE computations on the finer mesh. 


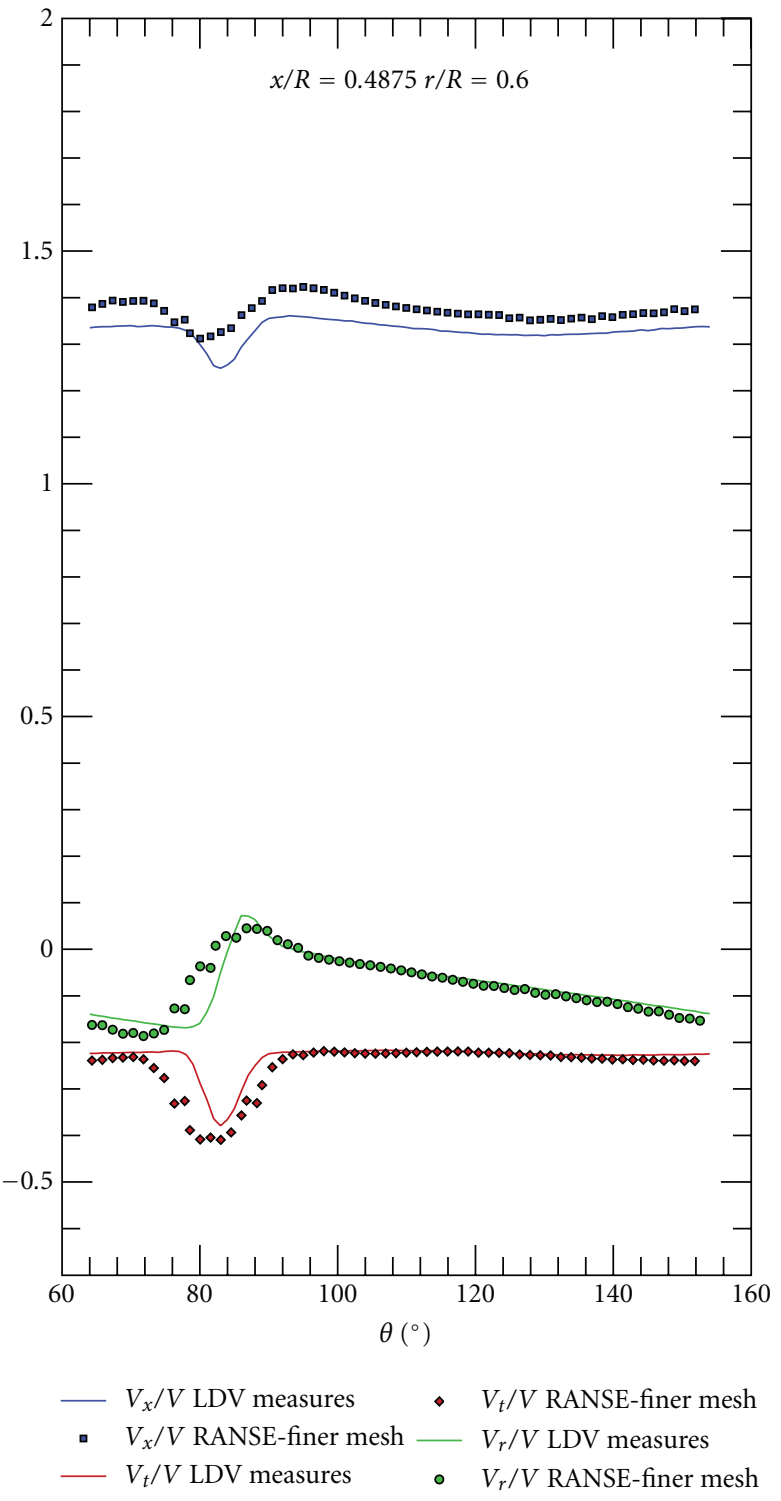

(a)

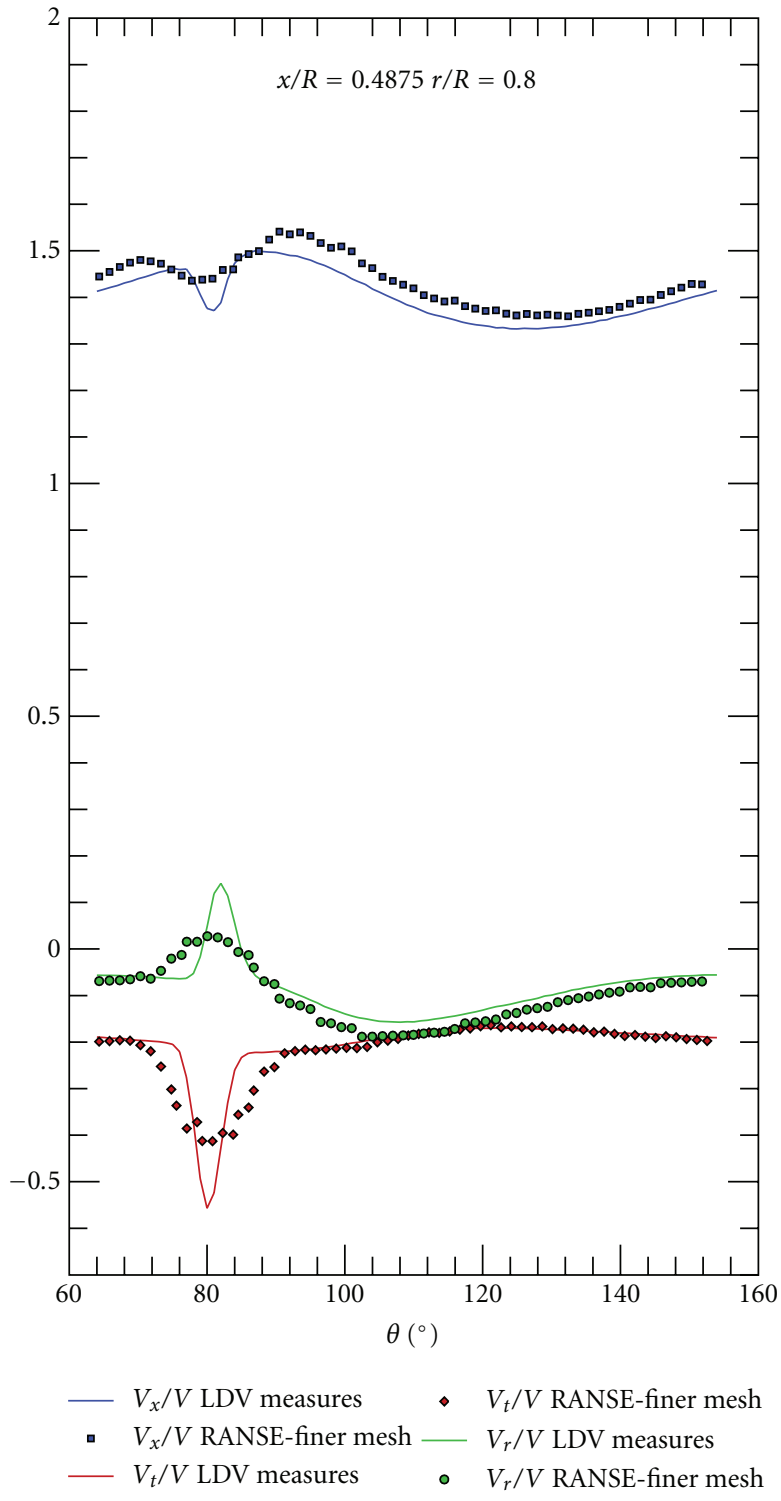

(b)

FIGURE 15: Axial, tangential, and radial vorticity distributions at $x / R=0.4875, r / R=0.60$, and $r / R=0.80$. Comparison between LDV and RANSE (single-blade angular sector).

a dedicated experimental campaign would be necessary to analyze possible limitations of the numerical approaches in this range. Cavitation tunnel results show, as usual, a slight shift with respect to open water tests at towing tank (the ratio between advance coefficients at same $K_{T}$ is about 0.985 ). This discrepancy is deemed acceptable considering differences between towing tank and cavitation tunnel environment; reference curves for comparison with numerical result in open water condition are those obtained at towing tank. LDV measurements and cavitation tests at the cavitation tunnel were carried out at fixed $K_{T}$ (thrust identity).

5.2. Propeller Wake. The complex flow structures generated by the endplate at the tip of the CLT propeller have been investigated by means of Laser Doppler Velocimetry, and measures have been compared with RANSE computations carried with the 8 million cells per blade mesh (to minimize numerical damping, as in Figure 7). Axial, tangential, and radial velocity distributions on ten planes (Table 1) were measured, but only four stations $(-0.25,0.4875,0.7$, and 0.9875 nondimensional position with respect to the propeller plane) are reported, together with the corresponding numerical computations. Vorticity results are compared with the numerical computations for the station at $x / R=0.5$. Differences between experimental measures and numerical computations, highlighted for the open water case of Figure 12, have been minimized performing the comparison with the thrust identity approach, therefore, assuming that the propeller wake field (within the cavitation tunnel during experiments or unbounded for the numerical calculations) 


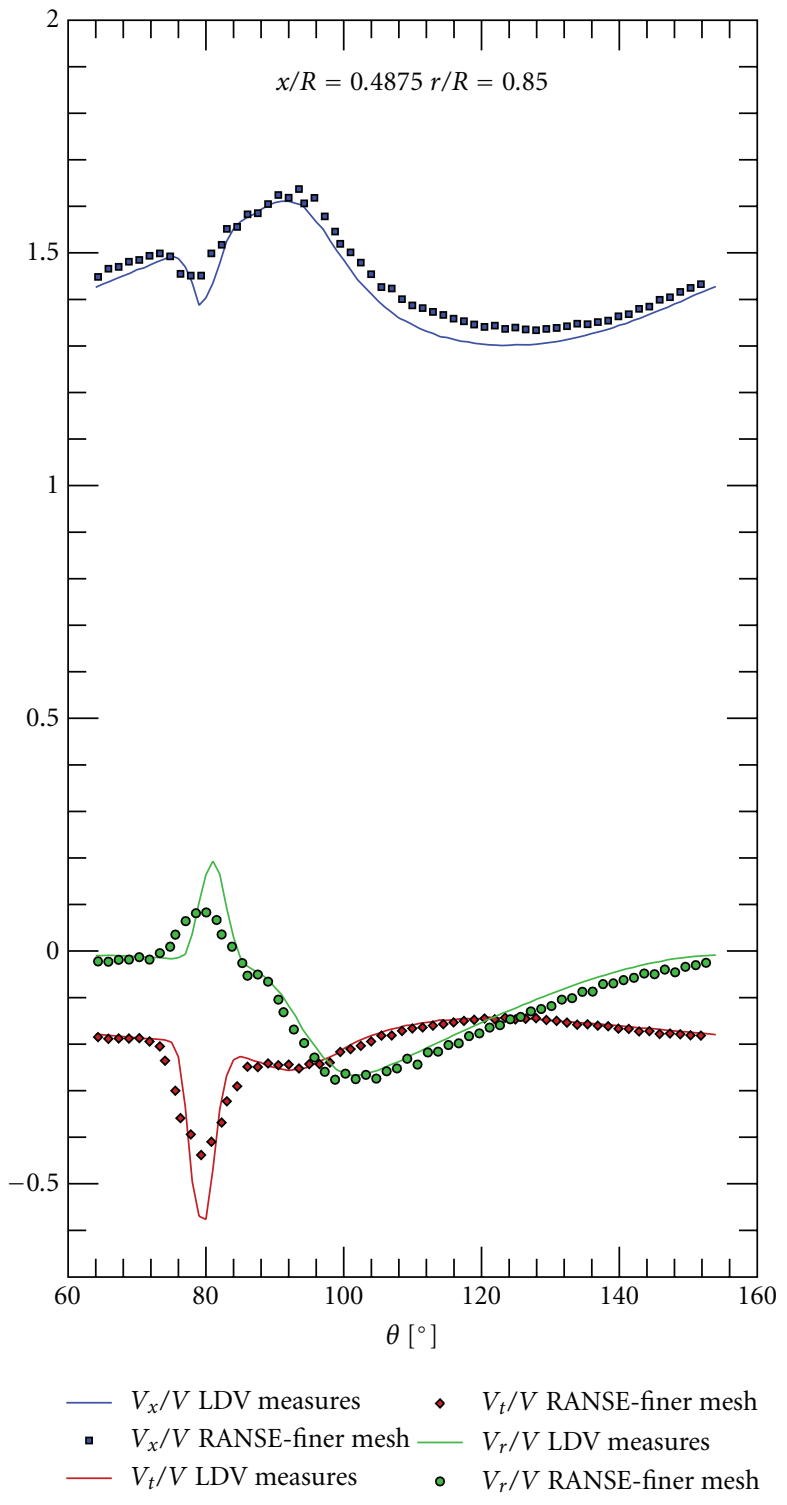

(a)

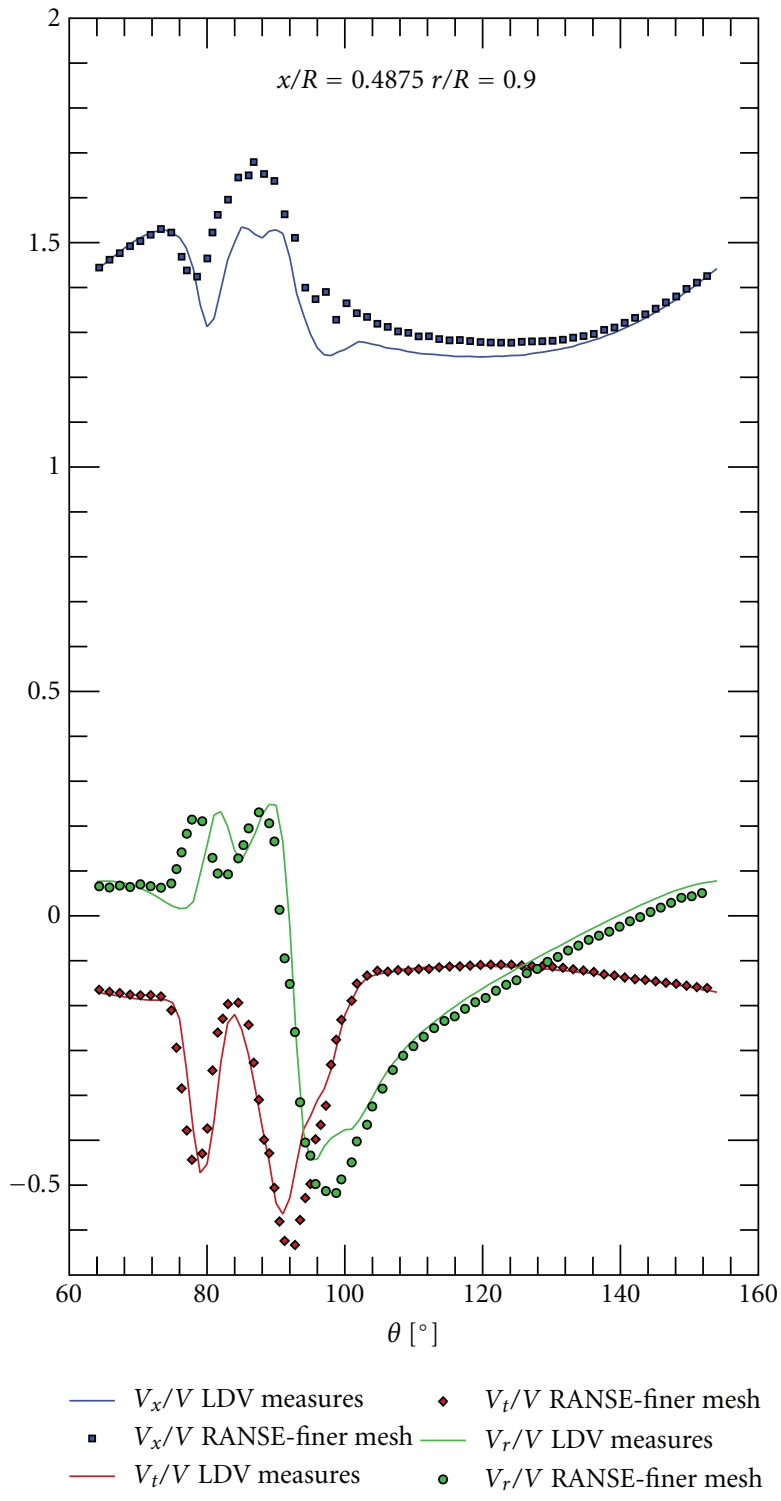

(b)

FIgure 16: Axial, tangential, and radial vorticity distributions at $x / R=0.4875, r / R=0.85$, and $r / R=0.90$. Comparison between LDV and RANSE (single blade angular sector).

behaves in the same way if the propeller delivers the same thrust. All the measures and the numerical computations, consequently, are referred to a thrust coefficient of 0.213 . Tests were carried out with propeller running at $18 \mathrm{~Hz}$, in order to avoid cavitation. All values presented are made nondimensional with respect to the undisturbed flow speed.

All the used representations of velocity distributions will be organized in the way to show the experimental results in the left column and the computational ones in the right one, in order to allow a constant comparison between measures and CFD results. The incoming flow in section $x / R=0.25$ (upstream of the propeller) is depicted in Figure 13, and for the sake of brevity, only the tangential component has been shown. The flow in this section is accelerating and slightly turning in order to enter the blade passage. As it can be observed, computed values still show a certain smoothing effect, even adopting the finest mesh; particularly, slightly lower values of the local maxima and minima are obtained. This aspect, joined with a larger perturbed area, leads to velocity gradients less defined in the CFD results. Nevertheless, it is important to point out that these differences are rather small, while in the plot, the adopted velocity scale has been reduced in order to sort out these small discrepancies. In Figure 14, results for the section at $x / R \quad 0.4875$ aft the propeller plane are reported and can be used to define the typical features of the downstream flow. In all the three velocity components, blade wakes and tip vortices are clearly visible and, in particular, the wakes can be identified in radial strips where the axial component is reduced, if compared to the passage areas, while the 


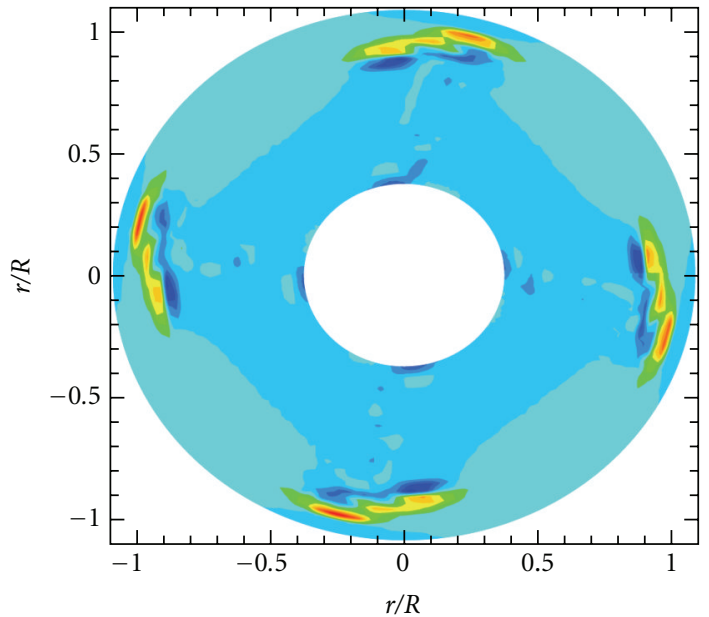

(a)

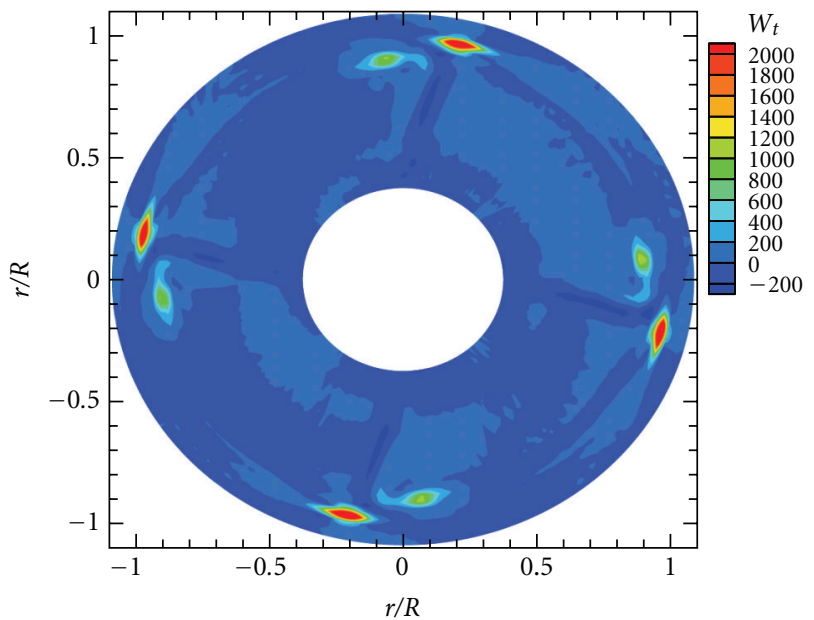

(c)

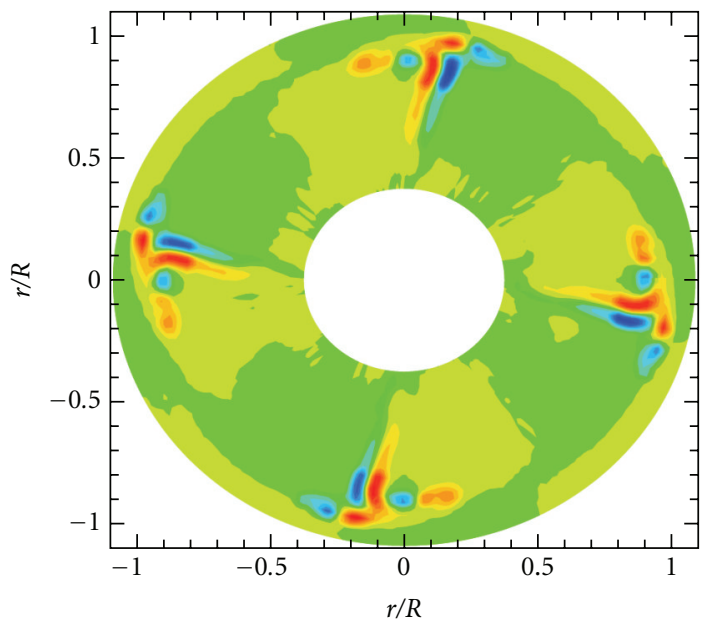

(e)

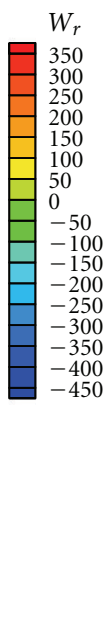

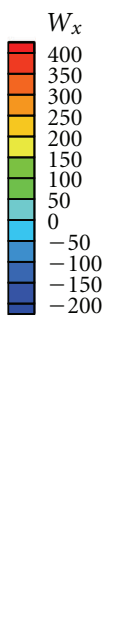
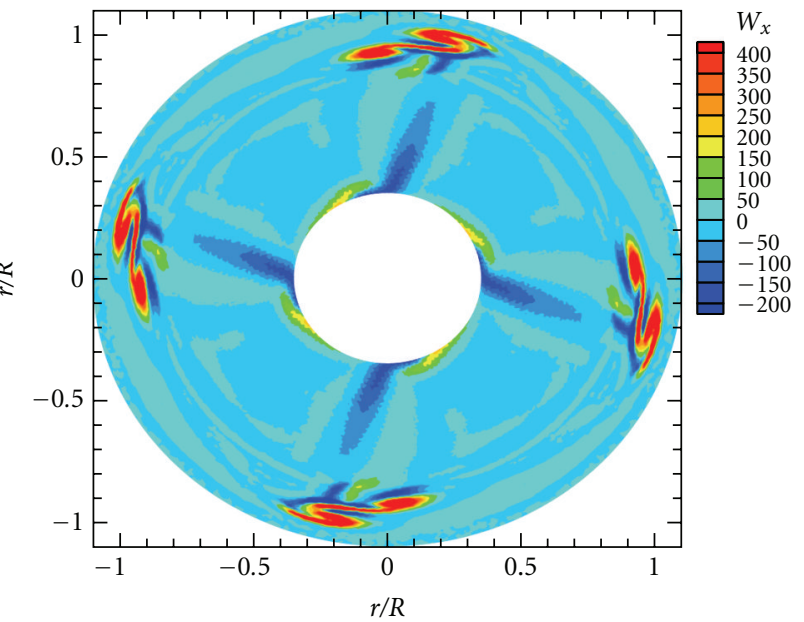

(b)

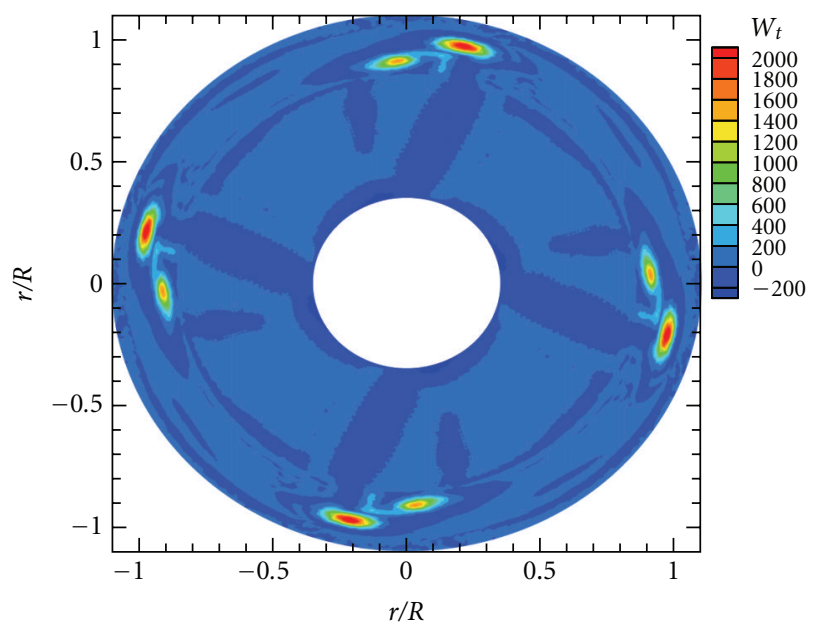

(d)

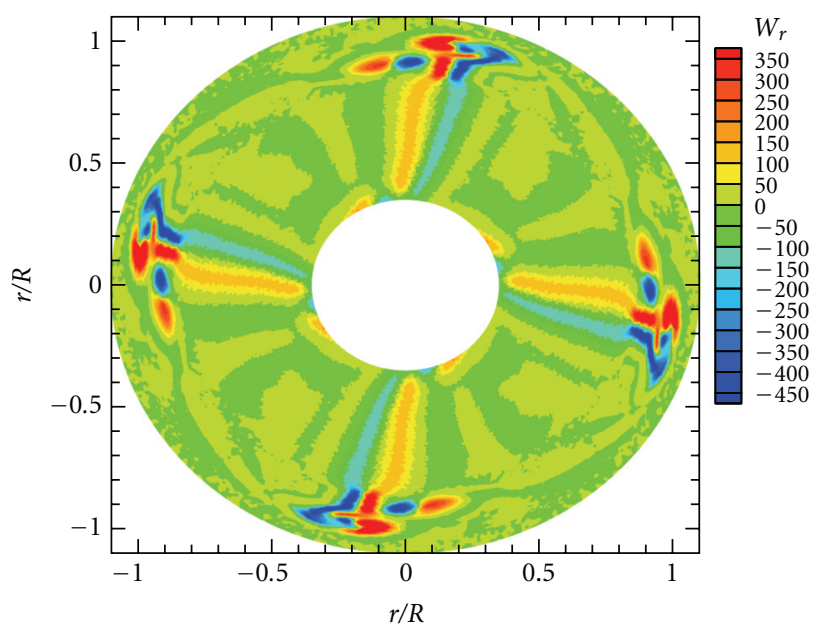

(f)

FIgURE 17: Axial, tangential, and radial vorticity distributions at $x / R=0.5000$ aft the propeller plane. ((a), (c), and (e)) Experimental LDV measurements. ((b), (d), and (f)) RANSE computations on the finer mesh. 


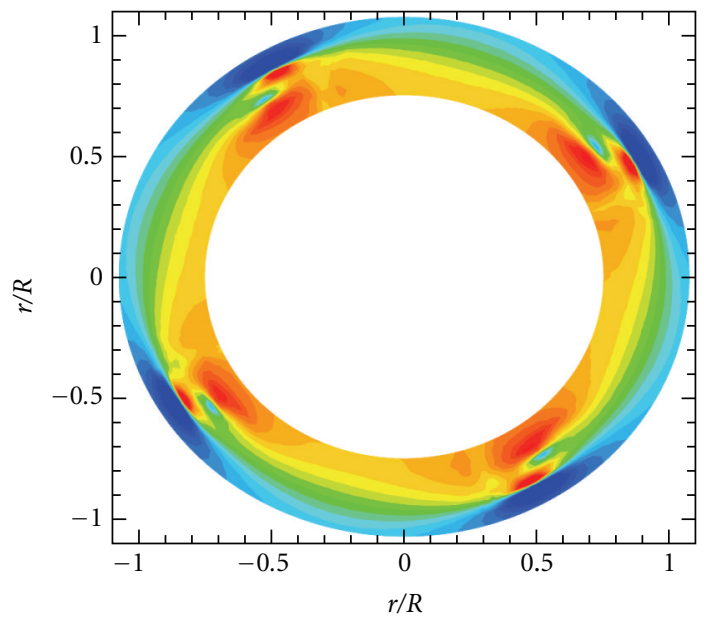

(a)

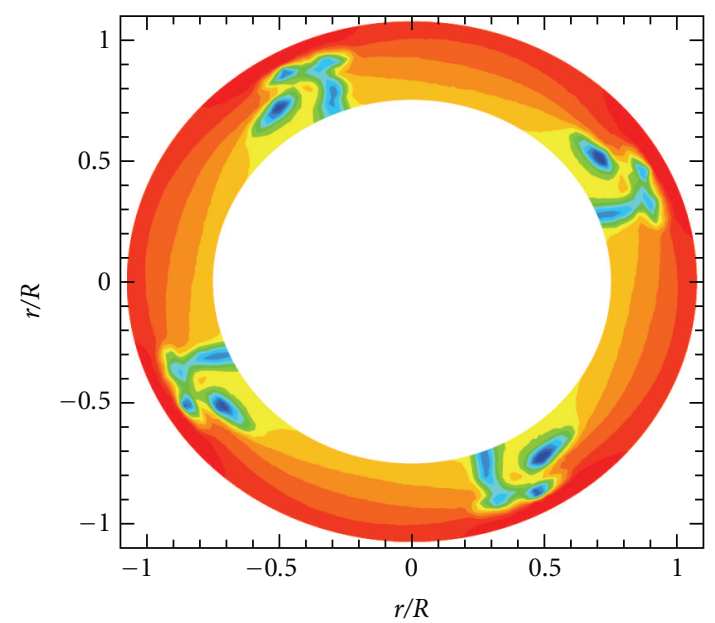

(c)

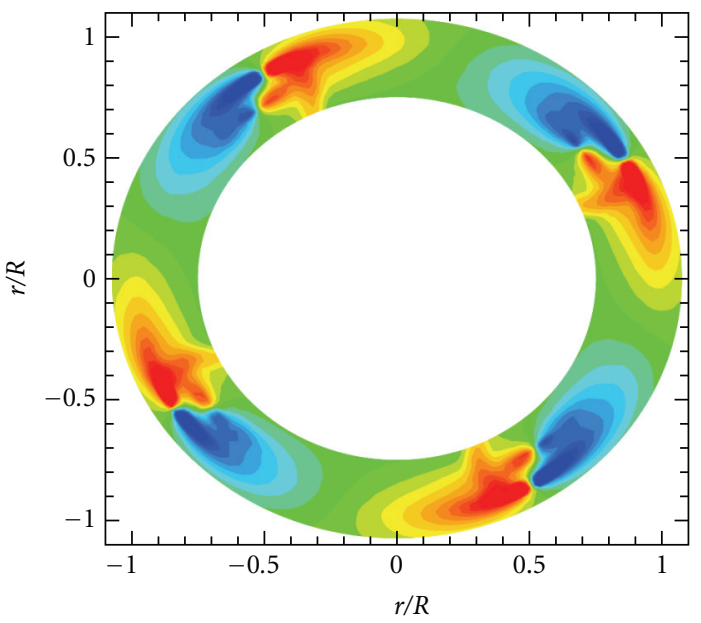

(e)
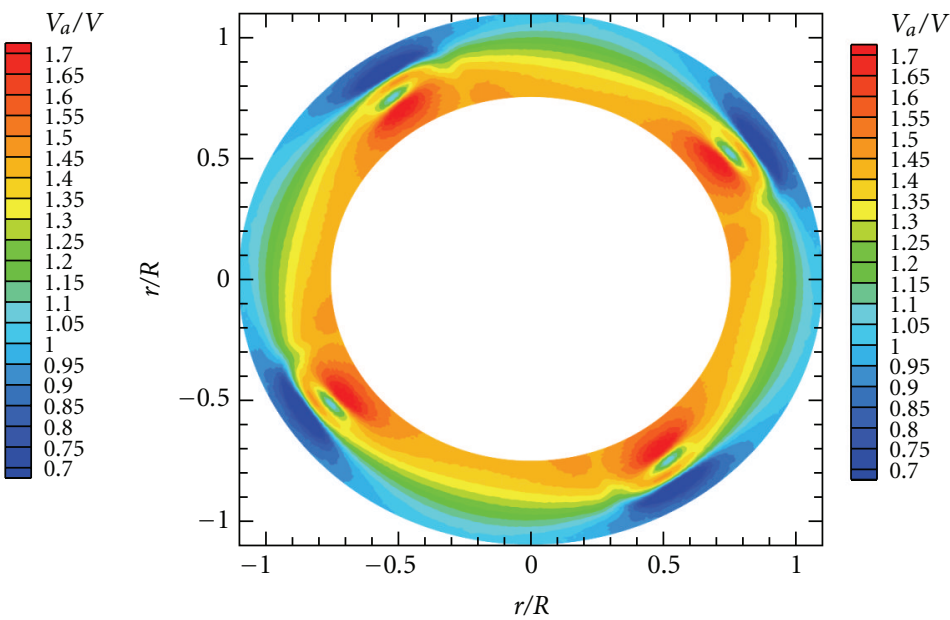

(b)

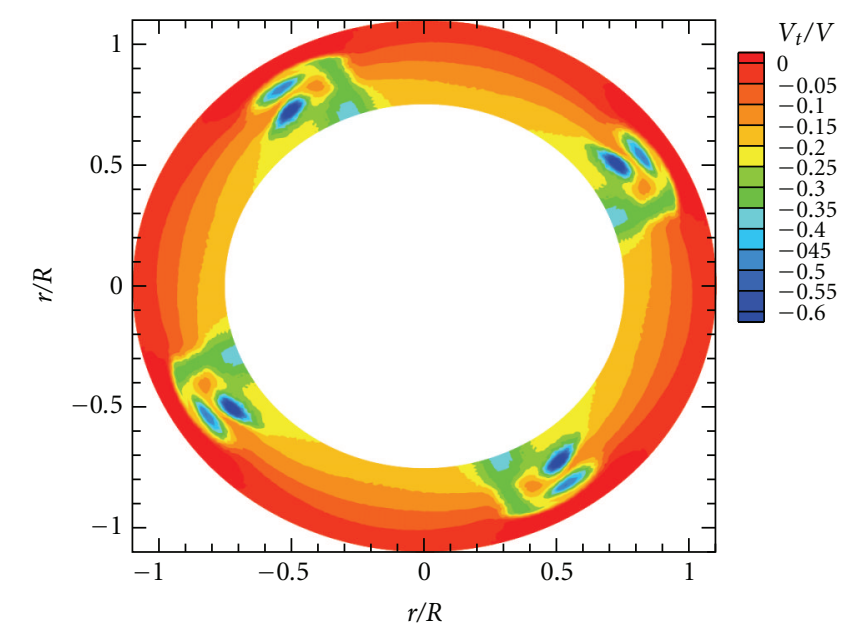

(d)
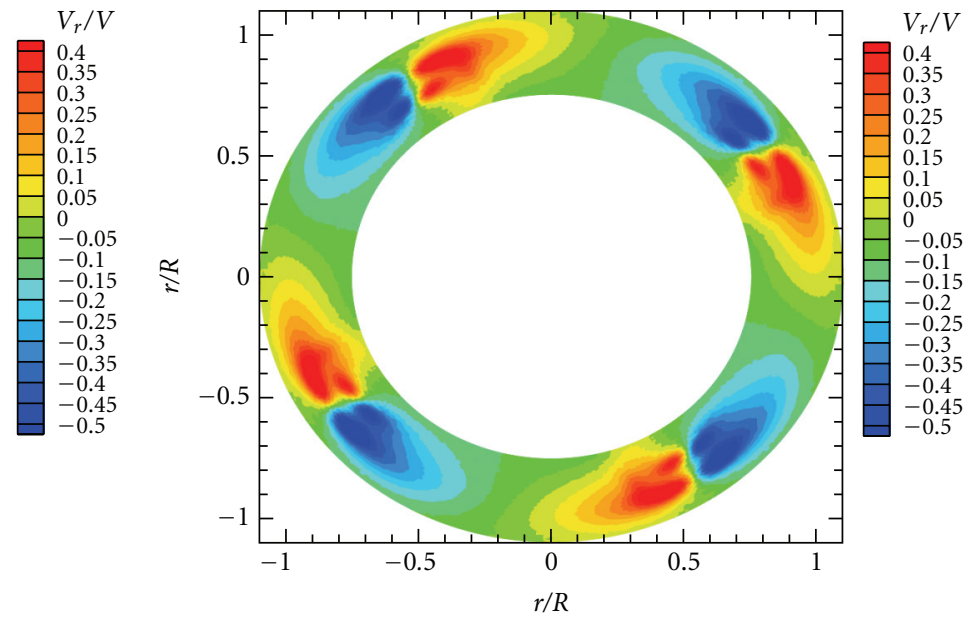

(f)

FigURE 18: Axial, tangential, and radial nondimensional velocity distributions at $x / R=0.7000$ aft the propeller plane. ((a), (c), and (e)) Experimental LDV measurements. ((b), (d), and (f)) RANSE computations on the finer mesh. 


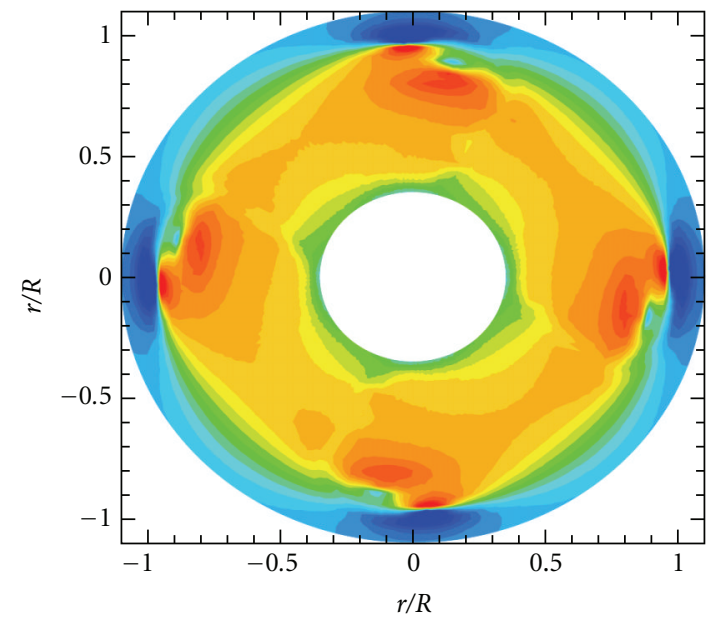

(a)

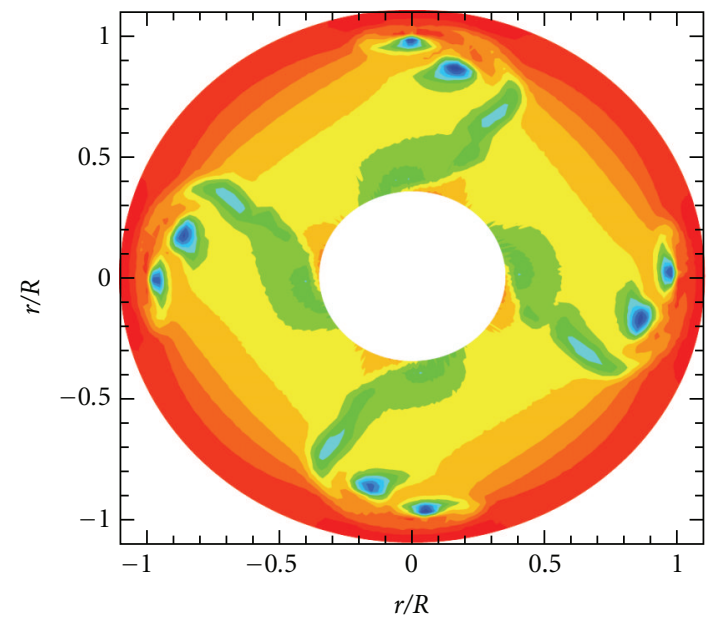

(c)

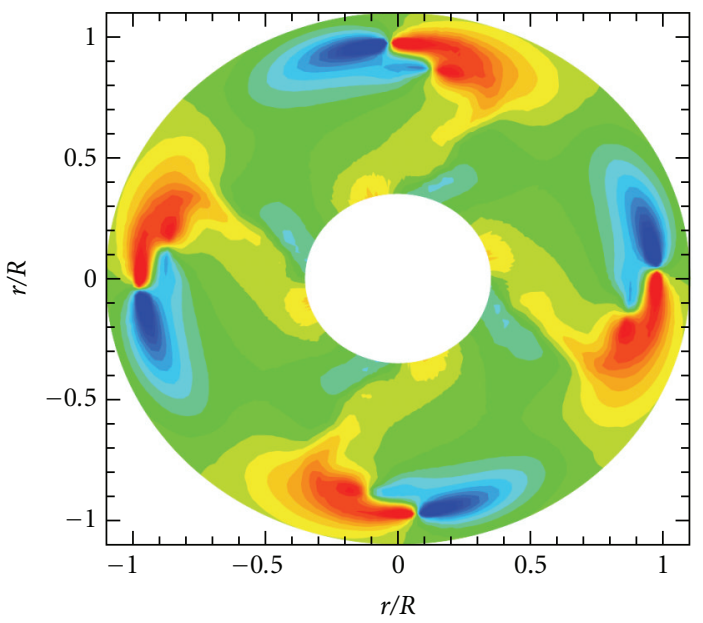

(e)
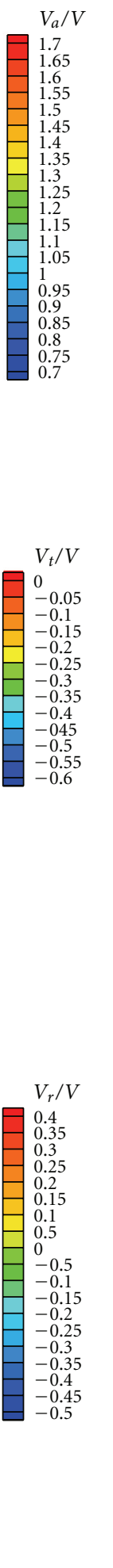
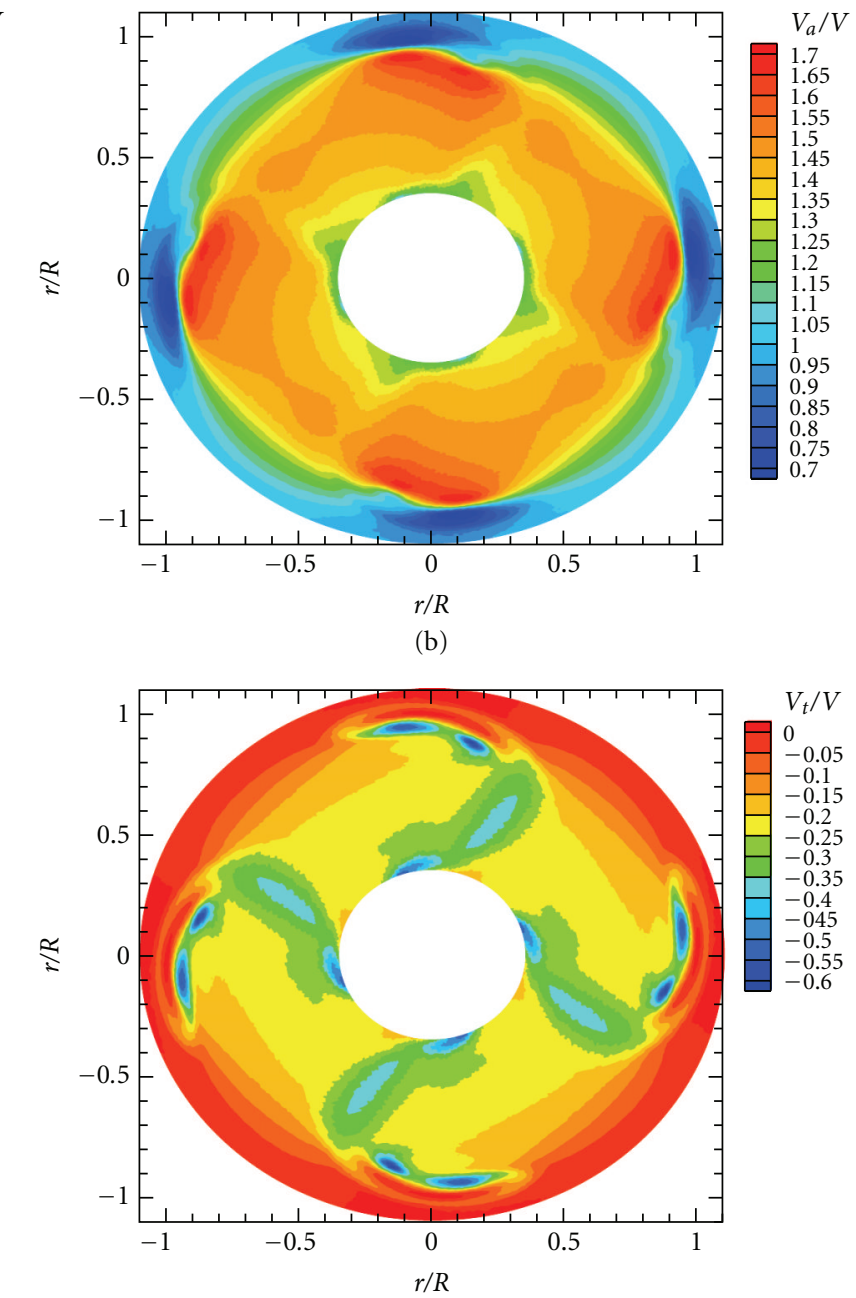

(b)

(d)

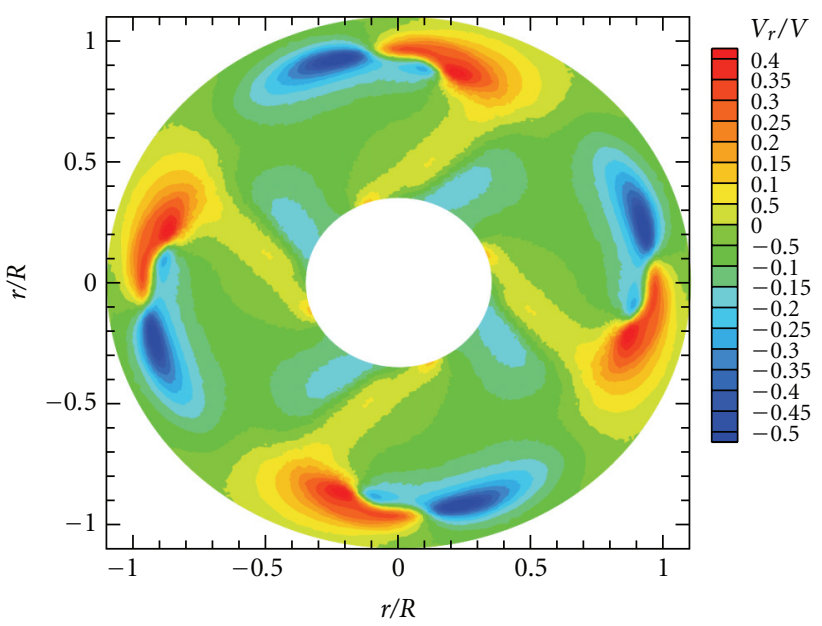

(f)

FIgURE 19: Axial, tangential, and radial nondimensional velocity distributions at $x / R=0.9875$ aft the propeller plane. ((a), (c), and (e)) Experimental LDV measurements. ((b), (d), and (f)) RANSE computations on the finer mesh. 
tangential one increases. Contemporarily, in the wakes, the radial component shows large values which are due to the stronger centrifugal force induced by the large tangential velocity component. It is interesting to underline the change in sign of the radial component through the wake which is present all along the blade span. Causes of this behavior can be found in the loading variations along the blade and in the presence of the tip vortices clearly visible at $r / R=$ 0.9. The presence of two vortical structures, which induce the largest velocity fluctuations in all the flow field, can be indifferently identified from the analysis of any of the velocity component distributions. In particular, one vortex is generated at endplate leading edge, where a geometrical discontinuity is present (successively named leading edge vortex), and propagates at a higher radius, while the second one (successively named blade tip vortex) is similar to the more usual tip vortex and propagates at a lower radius. Other remarkable flow characteristics are the reduced values of the axial and tangential components present in the outer flow, that is, the flow which has not passed through the propeller. The flow in this region has to slow down due to mass flow rate conservation and typically it is affected by slightly negative radial velocity. Differently in this section, the radial component distribution is largely perturbed by the presence of the strong vortical structures whose effects overcome the flow contraction. Even the inner flow region $(r / R=0.35)$ is affect, by low values of the axial velocity which are related to the presence of the hub boundary. Looking more in detail, the three velocity components, it is clear that the axial velocity presents a slightly higher acceleration in RANSE calculations (about 0.05-0.1 V), as it is also presented in Figure 15 for a series of radial sections. This difference may be partially due to the different operating conditions (in tunnel and in open water) even if no specific analysis/calculation has been carried out to confirm this. In addition to this, it is again evident that a certain degree of smoothing is present in RANSE calculations, clearly visible in the more diffused accelerated and decelerated flow areas in vortices region, and in less visible blade wake.

Similar considerations arise when tangential and radial velocities are considered. In this case, however, mean values of both velocities are correctly captured, with mean tangential velocity equal to about $0.2 \mathrm{~V}$ and a negative radial velocity indicating flow contraction. Local variations due to blade passage and to vortices are also captured, even if differences due to numerical smoothing are visible in the blade wake, which appears larger in space and slightly less pronounced in terms of absolute values (see also Figure 15 with values at $0.8 R$ and $0.85 R$ ). Regarding vortices, both strength (in terms of absolute velocities) and relative angular position seem correctly captured, as evidenced, qualitatively, in Figure 14 and, quantitatively, in Figure 16 with computed values at $0.9 R$. From this figure, the reasonable result of the computation of the successive peaks in velocities is evident, even if a slight angular shift and in some cases lower absolute values are present.

From all figures, it is evident that leading edge vortex has a higher strength, while blade tip vortex is weaker. Same

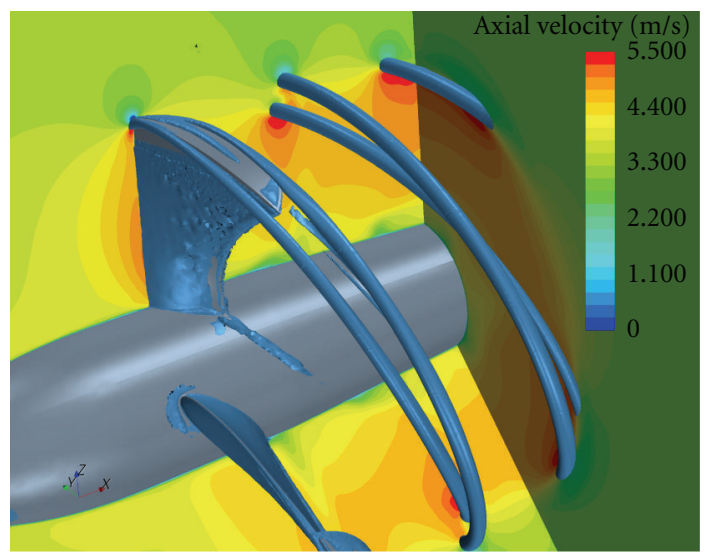

FIgURE 20: Vortical structures at the propeller tip. Q-factor visualization.

consideration arises also when propeller cavitating behavior is analyzed (Figure 21).

In Figure 17, axial, tangential, and circumferential vorticities, at nearly same longitudinal position (evaluated using measurements at $0.4875 R$ and $0.5125 R$ ), are reported. In this case, it is clear that, considering the most important case of tangential vorticity (tangential direction is the most similar to vortex propagation direction), computed values are very similar to measured ones, especially for the stronger leading edge vortex, with slightly higher values for the blade tip vortex. Other vorticity components show again a good agreement, with slightly higher computed values. An interesting aspect can be seen in the radial vorticity distribution in blade wake area, where a change of sign is present, due to the presence of the wake shear layer. Looking at sections more downstream the propeller (Figures 18 and 19), considerations similar to those already presented for section at $0.4875 R$ may be made. In particular, RANSE calculations tendency to smooth sharp variations in the flow field is clear again, with larger blade wakes and vortices area, and lower maximum and minimum values. Nevertheless, differences still appear acceptable, and numerical calculations allow to capture some interesting phenomena which are typical of this propeller functioning, such as the different pitch of the two vortices, evidenced by the different relative angular positions of vortex cores at different longitudinal positions. In particular, leading edge vortex presents a lower pitch, as visible in Figure 20 and in cavitation observations. This allows to consider the adopted calculation setup an acceptable compromise between calculation accuracy and computational effort (current calculations took about 20 hours on a 12 cores CPU).

In Figure 20, that shows the Q-factor isosurfaces, vortical structures captured with the RANSE approach are, once more, clearly evidenced. The Q-factor, defined as the sum of the symmetric and antisymmetric part of the velocity gradient tensor, is a common scalar quantity usually adopted in order to visualize vortical structures. From the figure, both different vortices pitches and strengths (in terms of diameter of the isosurface plots obtained for a Q-factor value equal to 30000) are visible. 


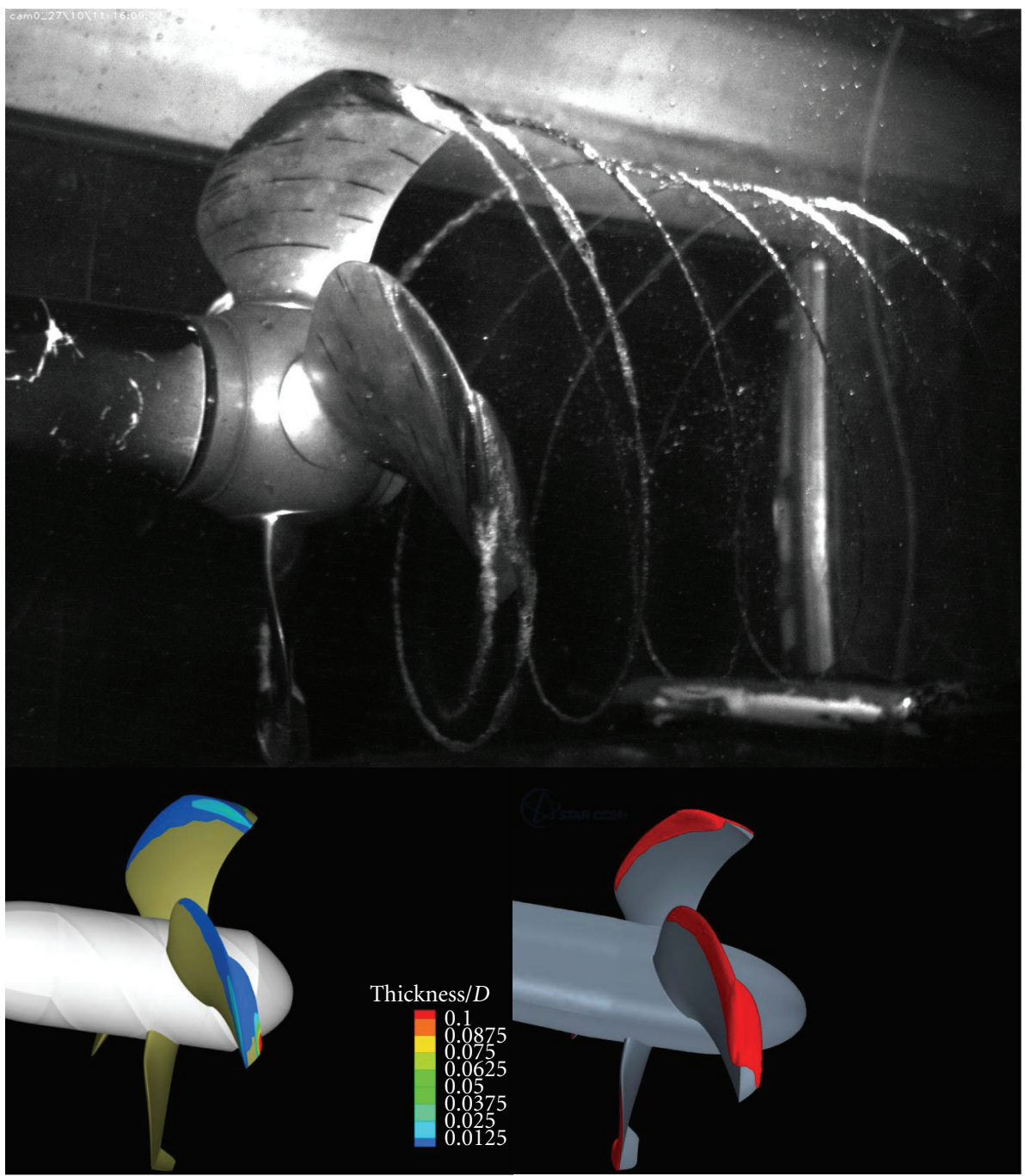

FIGURE 21: Cavity extension for open water KT equal to 0.213 , at cavitation index of 1.625 . Comparison with panel and RANSE numerical computations.

5.3. Cavitating Condition. The prediction of cavity extension is another key aspect for CLT unconventional propellers. The higher load near the tip, if cavitation cannot be avoided, could stabilize cavitation itself, especially when the propeller operates in a spatial non uniform wake behind the hull. On the contrary, the longer chord at the propeller tip could locally reduce the load per unit area, reducing the risk of cavitation and the bubble extension. Moreover, the influence on the pressure field of the endplate, that, with respect to a conventional geometry, increases the overpressure on the pressure side and reduces the pressure drop on the suction side, helps to reduce the back cavity extension. Anyhow, the presence of the endplate increases the risk of cavity inception (at its root and tip), and this potential problem should be taken into account for a better propeller design.

Prediction of cavity extension is, therefore, a necessary step in understanding the flow dynamics around CLT propellers. A steady analysis performed with the panel method, that has already proven to be a reliable and sufficiently accurate approach to compute steady and unsteady cavitating flow on conventional geometries $[2,13]$, can provide a preliminary insight into these phenomena. Moreover, a RANSE analysis can further deepen the knowledge, from a computational point of view, of the phenomena, giving additional details in the preliminary design stage of unconventional propellers.

Five different operating points have been considered for the analysis of the cavitating performances of the CLT propeller. As for the evaluation of propeller wake, all the computations have been carried out with the thrust identity. The first analyzed point (Figure 21) is the design point, that corresponds to an open water thrust coefficient of 0.213 at a cavitation index of 1.625. Second and third points (Figure 22) have been measured at the same thrust coefficient but at lower (1.5) and higher (3.3) cavitation indexes. The last two points (Figure 23), instead, are characterized by the design cavitation index (1.625) at lower (0.173) and higher (0.253) thrust coefficient. All tests at cavitation tunnel were carried out at $25 \mathrm{~Hz}$. 


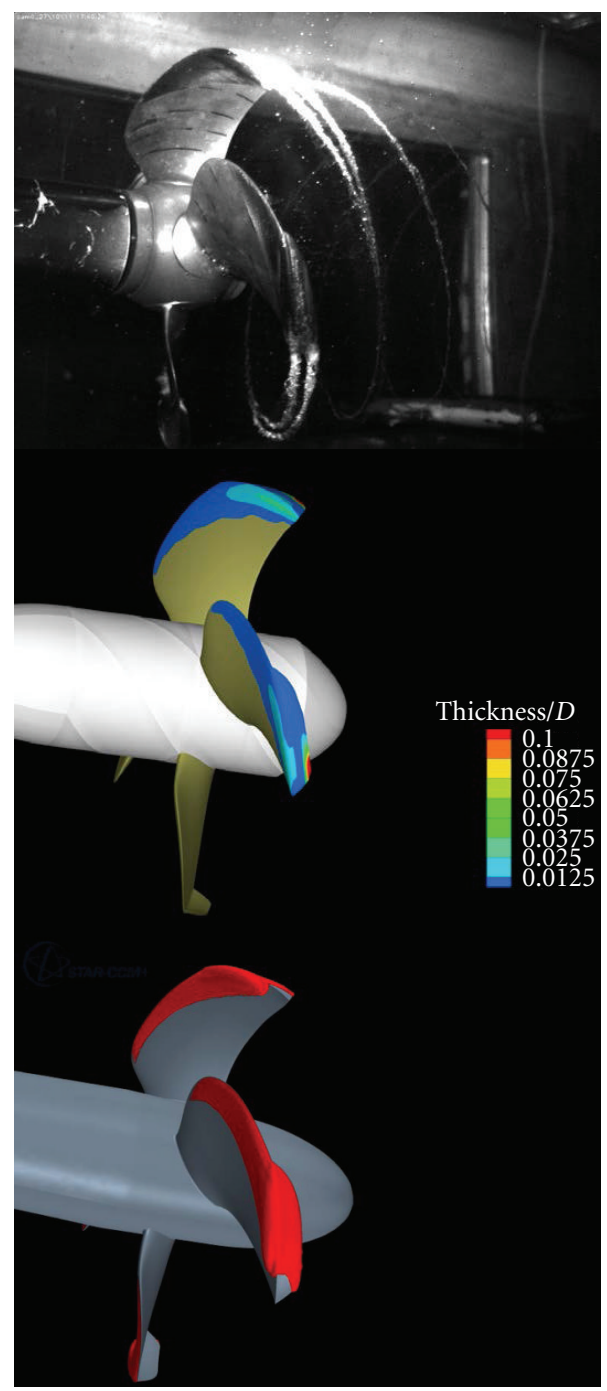

(a)

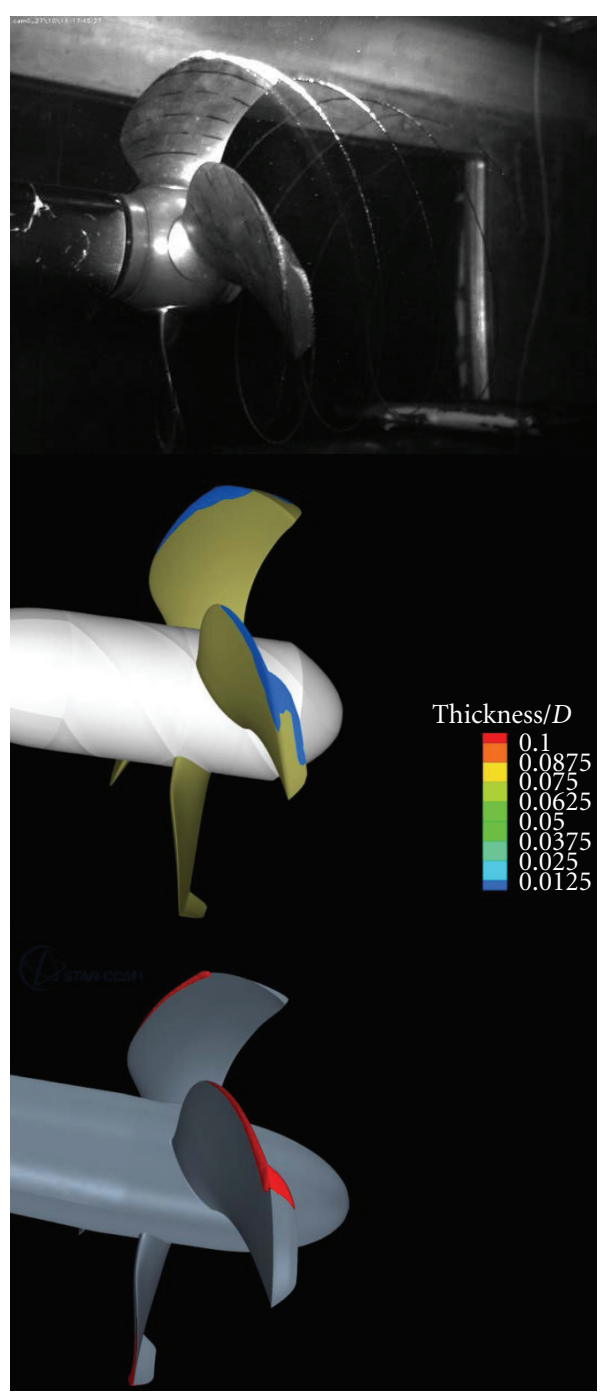

(b)

FIGURE 22: Cavity extension for open water KT equal to 0.213 , at cavitation index of 1.5 (a) and 3.3 (b). Comparison with panel and RANSE numerical computations.

From the experimental point of view it is possible to identify, first of all, the complexity of the vortical structures generated by the endplate. Both vortices cavitate, letting their visualization more immediate than the analysis of the velocity field downstream the propeller. The two vortexes, as previously highlighted, have different pitches, with the blade tip vortex having the higher one, causing a relative rotation of one vortex with respect to the other.

From a numerical point of view, the main features of the sheet cavity, identified at the cavitation tunnel, are captured quite well by the two solvers. In addition to the sheet cavitation on the suction side of the blade, the numerical computations predict the development of a sheet cavity bubble (from the leading edge) on the external side of the endplate, which results in a good qualitative agreement with the experimental evidence. Prediction of tip cavitating vortexes is beyond the capabilities of the developed panel method, that is, limited to the analysis of supercavitating sections. However, the thicker sheet cavity bubble at the blade trailing edge can be considered as a symptom of probable tip vortex cavitation. Especially at the lower advance coefficient (higher open water equivalent thrust) the prediction of sheet cavitation up to the trailing edge agrees well with the observed cavitating vortexes. RANSE computation could, in principle, take into account these phenomena. However, the numerical damping and dissipation, due mainly to an inadequate mesh for the computation of this kind of phenomena, drastically reduce the resolution at blade tip and only a thick sheet cavity is predicted at the endplate root, together with the sheet cavity at the endplate leading edge, well in agreement with the experimental observations.

The numerical predictions, anyway, can be considered satisfactory for assessing the cavitation properties of the propeller. Both the approaches well predict the influence of the cavitation index and of the advance/thrust coefficient. For almost all the considered conditions, the panel 


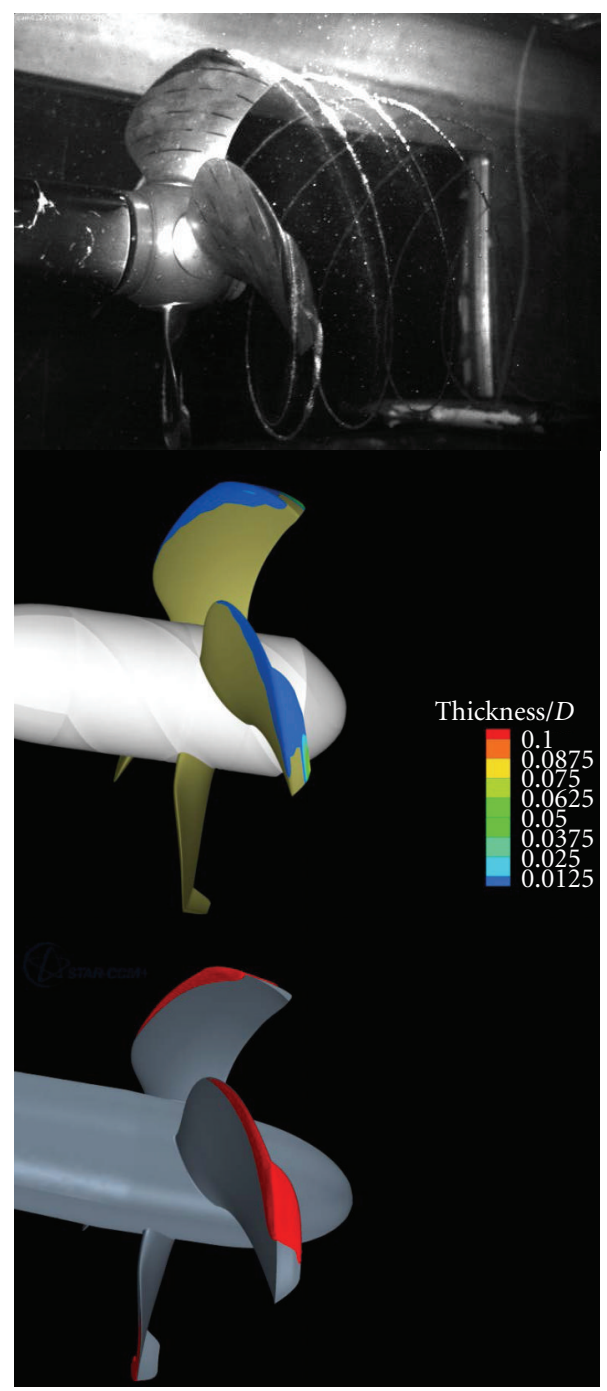

(a)

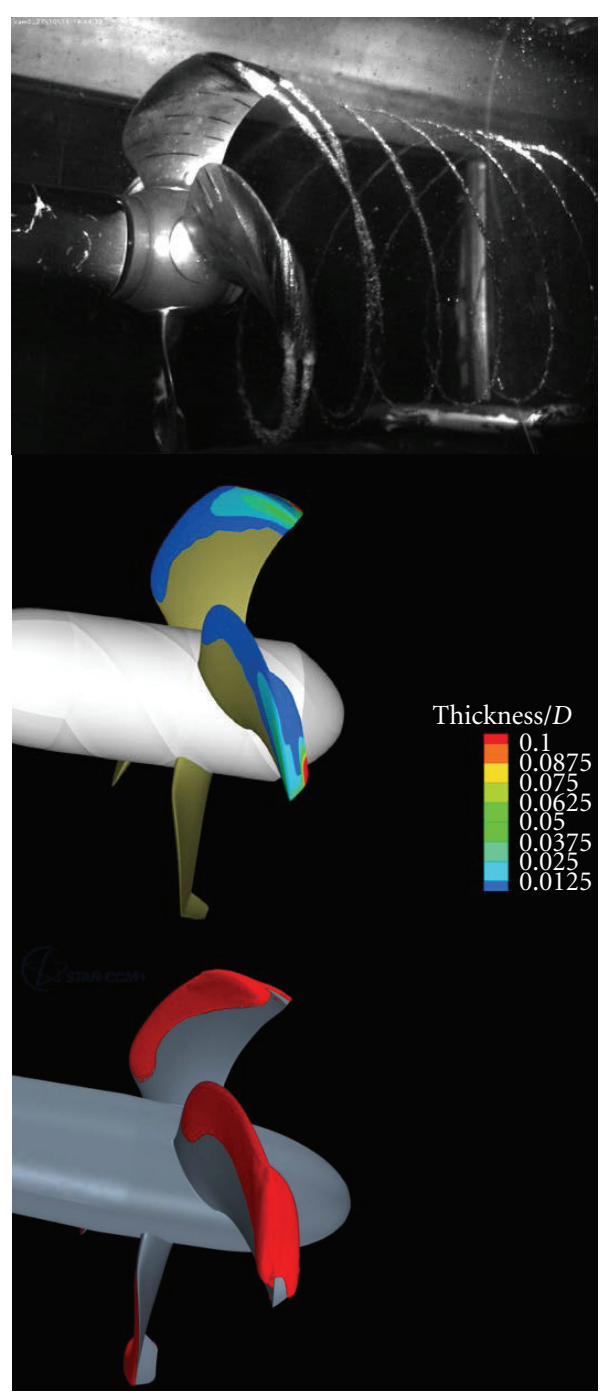

(b)

FIGURE 23: Cavity extension for open water KT equal to 0.173 (a) and 0.253 (b), at cavitation index of 1.625. Comparison with panel and RANSE numerical computations.

method, seems to overestimate the suction side cavity bubble extension, with respect to the RANSE solver, in chordwise directions, especially at the tip, while sheet cavity lengths predicted by RANSE are more extended in the radial direction. On the endplate predicted panel method sheet cavity bubble is longer, especially at the endplate root, and overlap all the outer endplate surface for higher thrust coefficients/lower cavitation indexes, while RANSE computations are, in general, more conservative (and more similar to observations). Altogether, panel method and RANSE are very close each other.

With respect to the observed cavity extensions, and except the cavitating vortexes, both the numerical predictions are a bit overestimated, mainly along the radial direction. This difference is probably due to the observed streak cavitation [20] starting atleading edge, and to the impossibility of differentiating it from sheet cavitation in both numerical methods. At the design point (Figure 21), for instance, sheet cavity bubble is observed starting from about $r / R$ equal to 0.8 . Numerical computations, instead, predict a very chordwisely short sheet bubble even around $r / R$ equal to 0.67 , where experimental observations highlight only streak cavitation with a more pronounced chordwise extension. Same considerations hold for lower cavitation indexes (Figure 22(a)) and higher loads (Figure 23(b)), with a predicted sheet cavity from $r / R=0.55$ versus $r / R=$ 0.70 experimentally observed. At sheet cavity inception (cavitation index of 3.3 for the thrust coefficient of 0.213 ) and for lower thrust coefficient (resp. Figure 22(b), and Figure 23(a)) only a slightly overestimated back sheet cavity bubble is predicted and also the different thickness/extension of the cavity bubble at endplate root and tip is representative of the less strong blade tip vortex with respect to the leading edge one. 


\section{Conclusions}

An extensive campaign at cavitation tunnel has been carried to measure equivalent open water performances, propeller wake characteristics, and cavitation of a nonconventional CLT propeller. At the same time, two numerical approaches, a potential panel method and a RANSE solver, have been successfully applied for the correspondent numerical analysis. Numerical computations of open water model scale characteristics at the design advance coefficient are in good agreement with experimental results, with negligible differences (thrust underestimated by $1.45 \%$ in the case of the panel method, $4.3 \%$ in the case of RANSE while torque coefficient is underestimated by $1.4 \%$ and overestimated by $1.5 \%$ in case of RANSE solver and panel method resp.). In off-design conditions, an overall good agreement has been achieved, with panel method results very close to towing tank measurements in the case of thrust (with a mean difference of $-1 \%$ in the considered range) and presenting a slight overestimation (about $1.3 \%$ as a mean) in the case of torque. Similarly, RANSE computations are satisfactory, with only weak underestimation of both coefficients (about $4 \%$ and $1.6 \%$ for thrust and torque coefficients resp.) for all the advance coefficients under investigation.

The LDV analysis highlighted the particular vortical wake structures associated with the endplate. The interaction between two vortical structures has been emphasized: the leading edge vortex and the blade tip vortex propagate downstream with different pitches. Also, in this case, the comparison with measures reveals that numerical computations are in good agreement for what regard the location, pitch, and, in general, the strength of the propeller wake. The sharp flow features are captured with sufficient reliability only with the finer mesh. Additional refinements seem to be necessary in the whole wake region (not only at the tip) to further minimize artificial smoothing (especially far from the propeller) and better identify flow features.

Finally, preliminary, steady, computations of the cavitating flow demonstrate that both panel method and RANSE solver can be employed, in a preliminary phase, and with sufficient accuracy, for the analysis of the cavitating behavior of these unconventional Tip Loaded Propellers. The main pattern of sheet cavitation (of sheet type at the endplate leading edge, cavitating vortexes at its edges) are predicted qualitatively and quantitatively quite well. A fine resolution of the RANSE discrete volume mesh is required for a good resolution at the tip and root of the endplate, in order to avoid smoothed solutions and to let the cavity bubble evolve also downstream into the fluid. These results allow to conclude, in any way, that the application of the potential panel method (which computational time is a fraction of that required by a viscous RANSE solver for the same flow conditions), in conjunction with a parametric geometry description and optimization algorithms may be used in an inverse design approach already demonstrated with success in case of conventional propellers [21, 22]. Further studies are planned in this directions by the authors.

\section{Acknowledgments}

This work was developed in the frame of the collaborative project SILENV-Ships oriented Innovative soLutions to rEduce Noise \& Vibrations, funded by the European Union. within the Call FP7-SST-2008-RTD-1 Grant Agreement SCP8-GA-2009-234182. The authors also would like to thank SISTEMAR and SINM and Dr. Enrique Haimov from CEHIPAR for their support and suggestions in carrying out these analyses.

\section{References}

[1] S. Pyo, Numerical modeling of propeller tip flows with wake sheet roll up in three dimensions [Ph.D. thesis], Massachusetts Institute of Technology, Cambridge, Mass, USA, 1996.

[2] S. Gaggero, Development of a potential panel method for the analysis of marine cavitating and supercavitating propellers [Ph.D. thesis], University of Genova, Genoa, Italy, 2010.

[3] D. Greeley, Marine propeller blade tip flows [Ph.D. thesis], Massachusetts Institute of Technology, Cambridge, Mass, USA, 1982.

[4] G. Dyne, "On the principles of propellers with endplates," International Journal of Maritime Engineering, vol. 147, no. 3, 2005.

[5] A. Sanchez-Caja, T. Sipila, and J. Pylkkanen, "Simulation of the incompressible viscous flow around an endplate propeller using a RANSE solver," in Proccedings of the 26th Symposium on Naval Hydrodynamics, Rome, Italy, 2006.

[6] S. Gaggero, S. Brizzolara et al., "Endplate effect propellers: a numerical overview," in Sustainable Maritime Transportation and Exploitation of Sea Resources, E. Rizzuto, S. C. Guede et al., Eds., CRC Press, Balkema, 2011.

[7] E. Canepa, A. Cattanei, M. Ferrando et al., "Studio sperimentale del flusso attorno ad un'elica per propulsione navale," in Proceedings of the Metodi di Sperimentazione delle Macchine (MIS MAC 7), Cagliari, Italy, April 2001.

[8] K. S. Min, "Numerical and experimental methods for the prediction of field point velocities around propeller blades," Tech. Rep., MIT Ocean Engineering Department, 1978.

[9] B. Lakshminarayana, "Techniques for aerodynamic and turbulence measurements in turbomachinery rotors," Journal of Engineering for Power, vol. 103, no. 2, pp. 374-392, 1981.

[10] A. Boutier, Accuracy of Laser Velocimetry, Lecture Series 199105, VKI, Brussels, Belgium, 1991.

[11] T. Strazisar, "Laser Anemometry in Compressors and Turbines," ASME Lecture on Fluid Dynamics of Turbomachinery, 1986.

[12] D. Modarress, H. Tan, and A. Nakayama, "Evaluation of signal processing techniques in laser anemometry," in Proceedings of the 4th International Symposium on Application of Laser Anemometry to Fluid Dynamics, Lisbon, Portugal, 1988.

[13] S. Gaggero and S. Brizzolara, "A panel method for transcavitating marine propellers," in Proceedings of the 7 th International Symposium on Cavitation, Ann Arbor, Michigan, Mich, USA, 2009.

[14] A. C. Mueller and S. A. Kinnas, "Propeller sheet cavitation predictions using a panel method," Journal of Fluids Engineering, vol. 121, no. 2, pp. 282-288, 1999.

[15] G. S. Hufford, Viscous flow around marine propellers using boundary layer strip theory [Ph.D. thesis], Massachusetts Institute of Technology, Cambridge, Mass, USA, 1992. 
[16] H. Curle, "A two parameter method For calculating the two dimensional incompressible laminar boundary layer," Journal of the Aeronautical Society, vol. 71, 1967.

[17] J. F. Nash and J. G. Hicks, "An integral method including the effect of upstream history on the turbulent shear stress for the computation of turbulent boundary layer," in Proceedings of the FOSR-IFT Stanford Conference, Stanfor University Press, 1969.

[18] CD-Adapco 2010, StarCCM+ v5 User's Manual, 2010.

[19] SILENV-Ship Oriented Innovative Solutions to reduce Noise and Vibrations, FP7 Collaborative Project 234182, Work Package 3, Subtask 3.1.1: Propellers, 2011.

[20] J. S. Carlton, Marine Propellers and Propulsion, ButterworthHeinemann, 2nd edition, 2007.

[21] D. Bertetta, S. Brizzolara, S. Gaggero, L. Savio, and M. Viviani, "Numerical and experimental characterization of a CP propeller unsteady cavitation at different pitch settings," in Proceedings of the 2nd International Symposium on Marine Propulsors, Hamburg, Germany, June, 2011.

[22] D. Bertetta, S. Brizzolara, S. Gaggero, L. Savio, and M. Viviani, "Numerical and experimental optimization of a CP propeller at different pitch settings," in Proceedings of the International Maritime Association of the Mediterranean, Genoa, Italy, September 2011. 

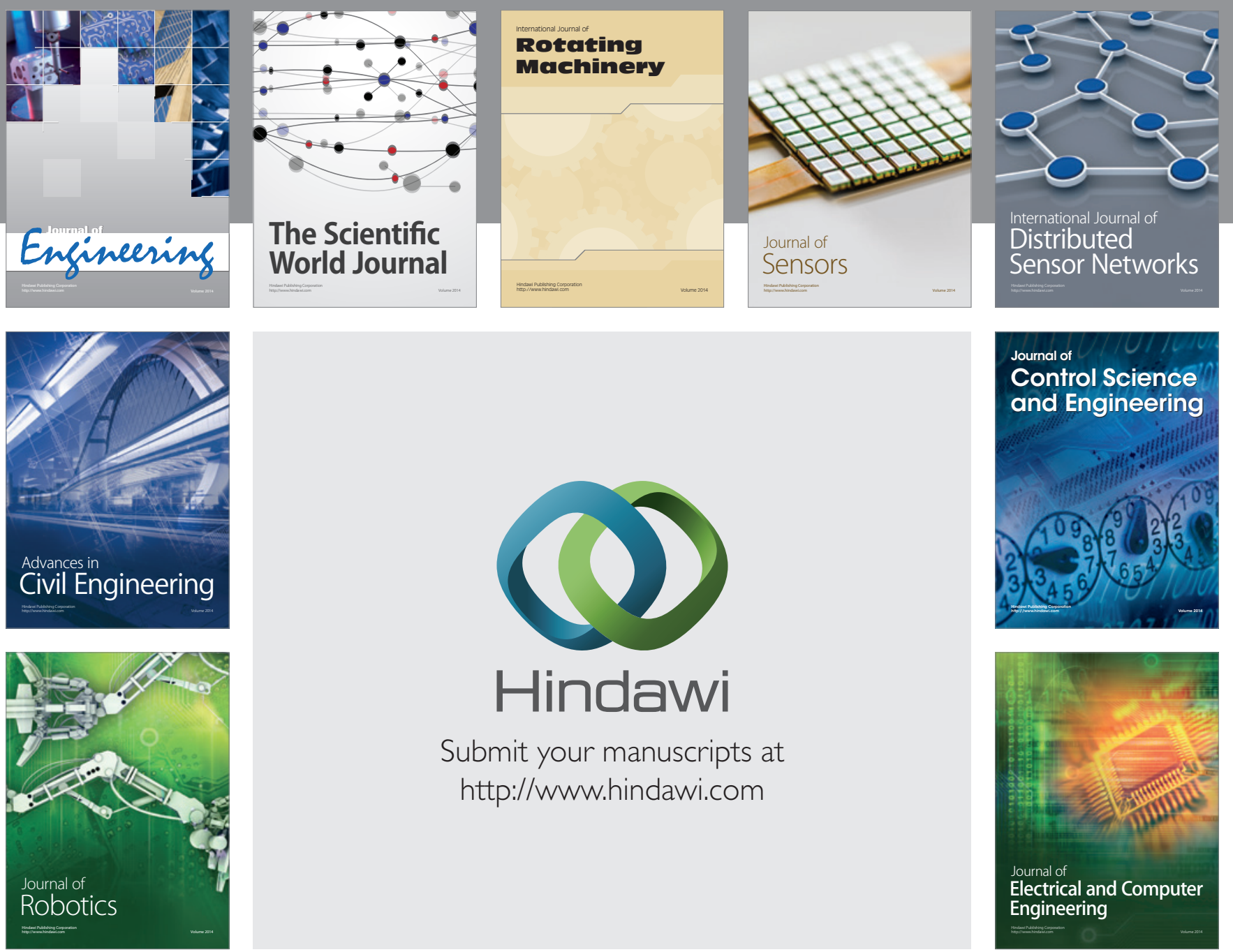

Submit your manuscripts at

http://www.hindawi.com
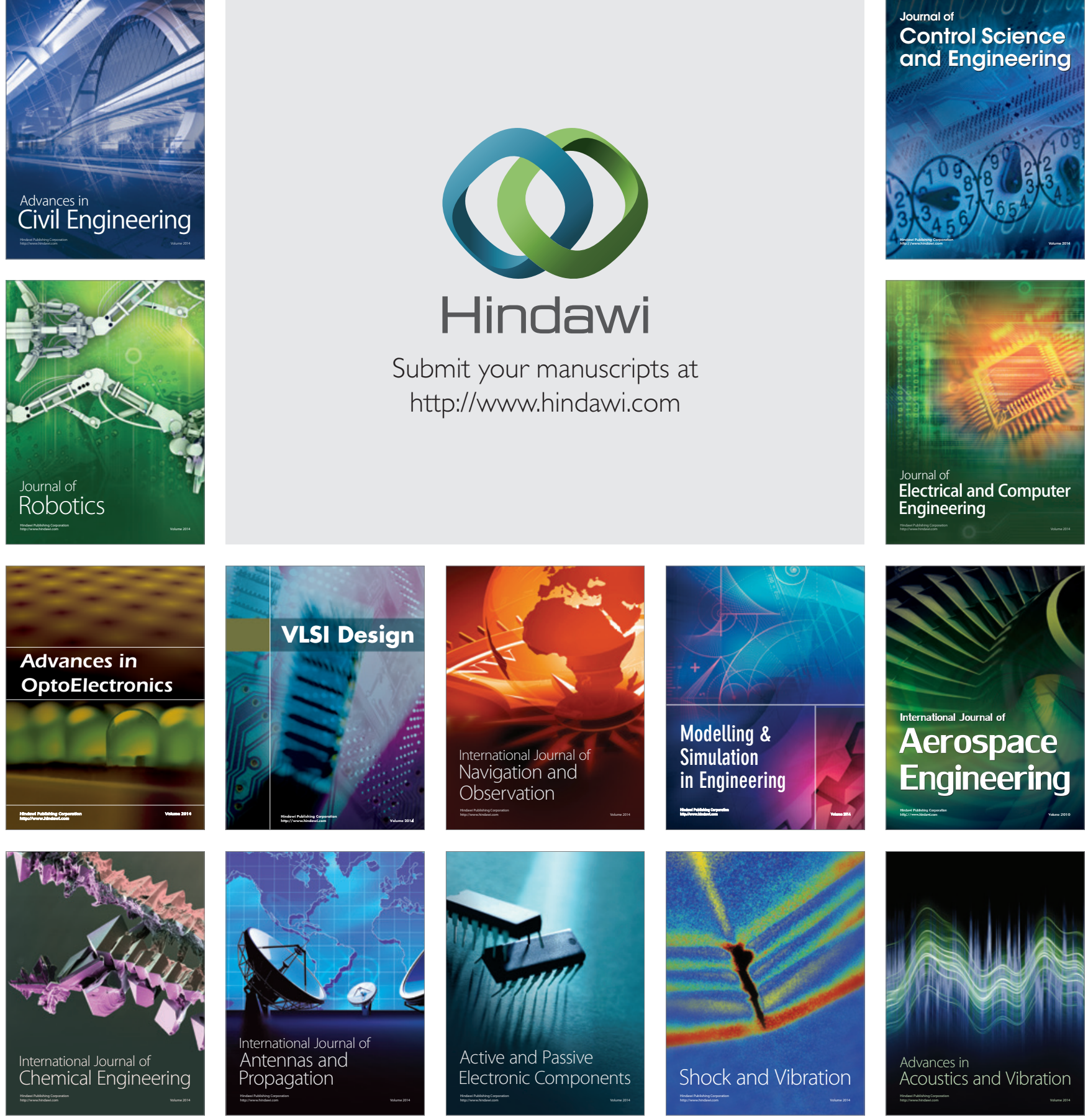